\title{
Gene therapy targeting survivin selectively induces pulmonary vascular apoptosis and reverses pulmonary arterial hypertension
}

\author{
M. Sean McMurtry, ${ }^{1}$ Stephen L. Archer, ${ }^{1}$ Dario C. Altieri, ${ }^{2}$ Sebastien Bonnet, ${ }^{1}$ Alois Haromy, ${ }^{1}$
} Gwyneth Harry, ${ }^{1}$ Sandra Bonnet, ${ }^{1}$ Lakshmi Puttagunta, ${ }^{3}$ and Evangelos D. Michelakis ${ }^{1}$

${ }^{1}$ The Vascular Biology Group and Pulmonary Hypertension Program, University of Alberta, Edmonton, Alberta, Canada. 2Department of Cancer Biology and the Cancer Center, University of Massachusetts Medical School, Worcester, Massachusetts, USA. ${ }^{3}$ Department of Pathology, University of Alberta, Edmonton, Alberta, Canada.

\begin{abstract}
Pulmonary arterial hypertension (PAH) is characterized by genetic and acquired abnormalities that suppress apoptosis and enhance cell proliferation in the vascular wall, including downregulation of the bone morphogenetic protein axis and voltage-gated $\mathrm{K}^{+}(\mathrm{Kv})$ channels. Survivin is an "inhibitor of apoptosis" protein, previously thought to be expressed primarily in cancer cells. We found that survivin was expressed in the pulmonary arteries (PAs) of 6 patients with PAH and rats with monocrotaline-induced PAH, but not in the PAs of 3 patients and rats without PAH. Gene therapy with inhalation of an adenovirus carrying a phosphorylationdeficient survivin mutant with dominant-negative properties reversed established monocrotaline-induced PAH and prolonged survival by $25 \%$. The survivin mutant lowered pulmonary vascular resistance, RV hypertrophy, and PA medial hypertrophy. Both in vitro and in vivo, inhibition of survivin induced PA smooth muscle cell apoptosis, decreased proliferation, depolarized mitochondria, caused efflux of cytochrome $c$ in the cytoplasm and translocation of apoptosis-inducing factor into the nucleus, and increased $\mathrm{Kv}$ channel current; the opposite effects were observed with gene transfer of WT survivin, both in vivo and in vitro. Inhibition of the inappropriate expression of survivin that accompanies human and experimental PAH is a novel therapeutic strategy that acts by inducing vascular mitochondria-dependent apoptosis.
\end{abstract}

\section{Introduction}

Pulmonary arterial hypertension (PAH) is a disease of the pulmonary vasculature, defined by an elevated pulmonary vascular resistance (PVR), which eventually leads to right heart failure and premature death. The cause remains unknown, and available treatments are limited, expensive, and often associated with significant side effects (1). In PAH, the pulmonary arteries (PAs) manifest pathological proliferative vascular remodeling that includes cellular proliferation in both the intima and the media and muscularization of the normally thin-walled distal PAs (1). Endothelial dysfunction results in an increase in the ratio of endothelial-derived vasoconstrictors to vasodilators. This imbalance has been the basis of therapies over the past several years; for example, exogenous delivery of vasodilating prostaglandins or blockade of the endothelin axis. However, less that $10 \%$ of the patients respond to selective pulmonary vasodilators. While vasoconstriction contributes, especially early in $\mathrm{PAH}$, the obstructive vascular remodeling is the major cause of the elevated PVR and ultimately the right heart failure, which in turn accounts for the $50 \% 5$-year mortality in this disease (1). There is now a shift in the interest of the scientific community, focusing on therapies aiming to reverse the proliferative remodeling in $\mathrm{PAH}(2)$.

Nonstandard abbreviations used: AIF, apoptosis-inducing factor; Kv, voltage-gated $\mathrm{K}^{+}$(channel); MCT, monocrotaline; MCT-PAH, MCT-induced pulmonary arterial hypertension; PA, pulmonary artery; PAAT, PA acceleration time; PAH, pulmonary arterial hypertension; PASMC, PA smooth muscle cell; PCNA, proliferating cell nuclear antigen; PVR, pulmonary vascular resistance; PVRi, PVR index; RVH, RV hypertrophy; TMRM, tetramethylrhodamine methyl-ester.

Conflict of interest: The authors have declared that no conflict of interest exists.

Citation for this article: J. Clin. Invest. 115:1479-1491 (2005).

doi:10.1172/JCI23203.
Several abnormalities that have been described in PAH contribute to a resistance to apoptosis and a proliferation/apoptosis imbalance within the vascular wall and might explain the PA remodeling: First, in a subset of $\mathrm{PAH}$ patients, germ-line and acquired loss-of-function mutations have been described in bone morphogenetic protein receptor 2 (BMPR2) $(3,4)$. Activation of the BMPR2 axis leads to suppression of proliferation and activation of apoptosis in normal PA SMCs (PASMCs) (5) but not in PASMCs from patients with $\mathrm{PAH}$ (6). Second, gene microarray studies show that in patients with $\mathrm{PAH}$, there is dysregulation of mediators of apoptosis in the PA wall that favors suppression of apoptosis. For example, bcl-2 is upregulated in PAH (7). Third, specific PASMC voltage-gated $\mathrm{K}^{+}(\mathrm{Kv})$ channels, such as $\mathrm{Kv} 1.5$, are downregulated in both animal models $(8,9)$ and human PAH $(10)$. By controlling the membrane potential in PASMCs (11), Kv channels regulate the opening of the voltage-gated L-type $\mathrm{Ca}^{++}$channels. When $\mathrm{Kv}$ channels are inhibited, the influx of $\mathrm{Ca}^{++}$causes both PASMC contraction and increased proliferation rates (12). This is further enhanced by the upregulation of transient receptor potential channel genes, which encode for $\mathrm{Ca}^{++}$channels during proliferation and were recently described in the PASMCs of patients with PAH (13). Furthermore, by blocking the egress of $\mathrm{K}^{+}$down its intracellular/ extracellular gradient (145:5 mEq), the Kv channel downregulation elevates intracellular $\mathrm{K}^{+}$. Because intracellular $\mathrm{K}^{+}$tonically inhibits caspases, the inhibition or lack of $\mathrm{Kv}$ channels contributes to a resistance to apoptosis (14).

Survivin, a member of the mammalian "inhibitor of apoptosis" family (15), is known to be expressed in essentially all cancers but not in most normal adult cell types (16). The cell cycle-dependent expression of the survivin gene in mitosis suggests a role 
A

A
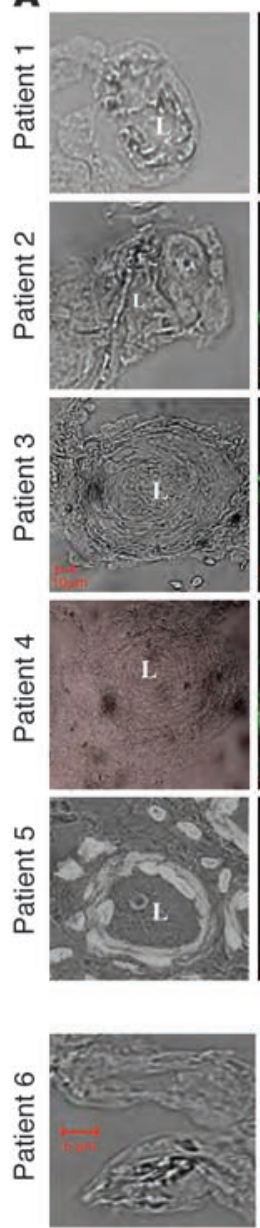

SMA
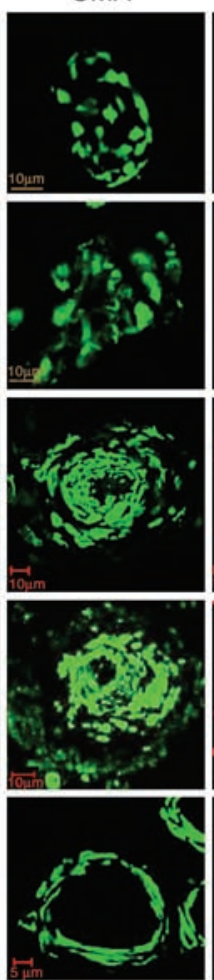

vWF

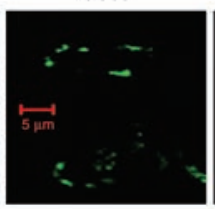

S
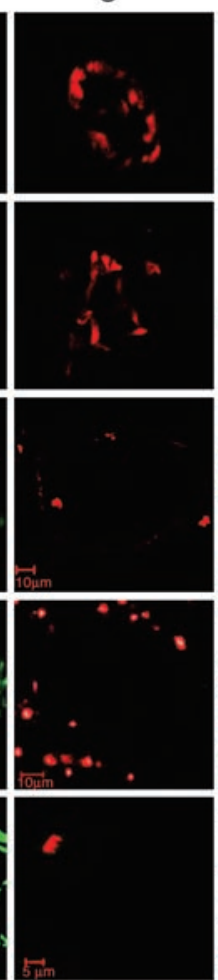

S

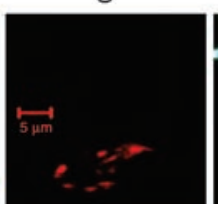

$\mathrm{SMA}+\mathrm{S}+\mathrm{DAPI}$
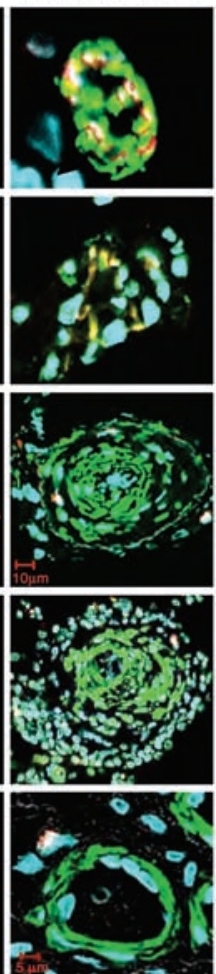

vWF+S+DAPI

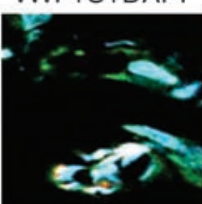

$\mathbf{B}$
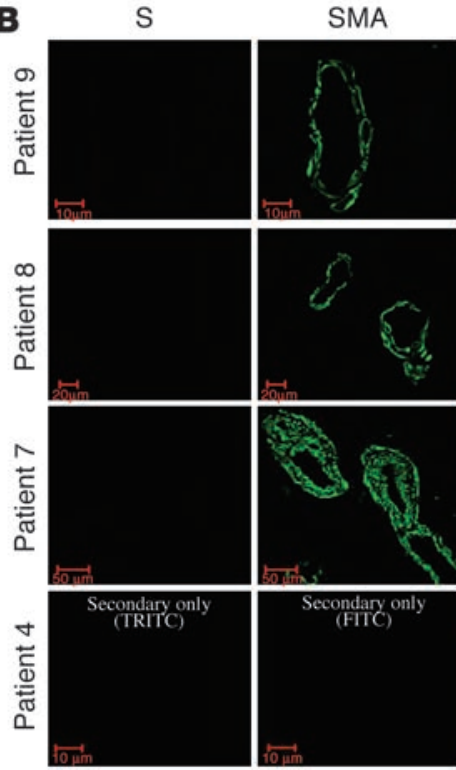

$\mathrm{SMA}+\mathrm{S}+\mathrm{DAPI}$
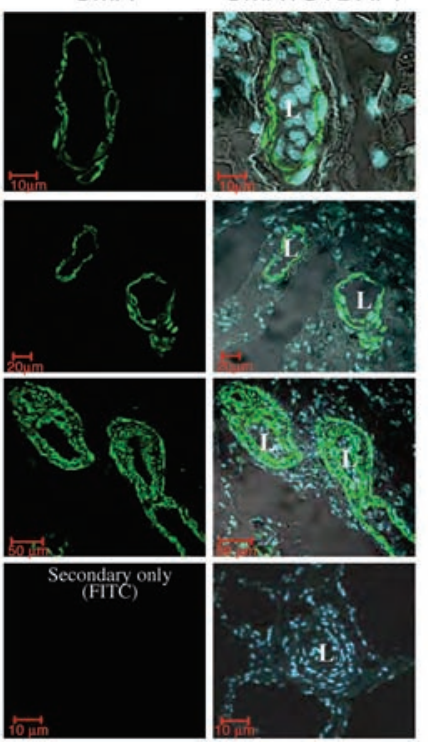

\section{Figure 1}

Survivin is expressed in the PAs of patients with PAH, but not the PAs of patients with secondary pulmonary hypertension or normal PAs. (A) Top: Immunofluorescence confocal microscopy shows survivin expression colocalizing with smooth muscle actin in the media of small and medium-sized PAs from 5 patients with PAH. In some cases (patients 3-5) survivin is expressed in cells both outside and inside the elastic lamina (evident by autofluorescence in patient 3). Bottom: In 2 small PAs shown from a PAH patient, survivin is also expressed; it colocalizes with vWF only in the bottom one, which appears to be more remodeled and have an almost obliterated lumen. (B) Survivin is not expressed in the PAs of 3 patients without PAH (patients 8 and 9; patient 10 not shown). Despite the significant medial hypertrophy shown in patient 7, due to thromboembolic pulmonary hypertension, there is a compete lack of survivin expression, suggesting that it is not a nonspecific feature of the remodeling process. Lack of nonspecific staining by the secondary antibodies in patient 4 is shown in the bottom; this was the case in all the patients shown here. SMA, smooth muscle actin; S, survivin; L, lumen.

for survivin in promoting cell proliferation; however, recent data point to a more selective role of survivin in antagonizing mitochondria-dependent apoptosis (reviewed in ref. 16), and a mitochondrial pool of survivin has recently been shown in cancer cells (17). The absence of survivin from most healthy tissues makes it very attractive as a target for therapy. Molecular antagonists of survivin, including antisense and dominant-negative mutants, have been consistently associated with induction of apoptosis and inhibition of tumor growth in vivo, without affecting normal cells (16). Such a mutant is the Thr34 $\rightarrow$ Ala (here described as survivin-M). This mutation prevents a critical phosphorylation of endogenous survivin by the mitotic kinase p34 ${ }^{\text {cdc2 }}-$ cyclin B1. Survivin-M has a 4- to 5-fold accelerated clearance compared with WT survivin and results in a dominant-negative effect by lowering endogenous survivin levels (16). In addition to inducing apoptosis in cancer cells (16), survivin targeting with survivin$\mathrm{M}$ prevents vascular remodeling in an arterial injury model by inducing apoptosis within the vascular wall (18). In that study, survivin was absent in quiescent SMCs but was induced in vitro by exposure of aortic SMCs to $20 \%$ serum or PDGF or selectively in injured arterial segments in vivo (18).

The pulmonary circulation is very different from the systemic circulation; for example, the pulmonary circulation has low pressure compared with the systemic circulation and constricts to hypoxia, while the systemic circulation dilates (19). This difference might be in part due to the fact that PASMC mitochondria, important oxygen sensors, are different from the systemic arterial SMC mitochondria: they have lower respiratory rates, are more depolarized, have more manganese superoxide dismutase (MnSOD), and produce more hydrogen peroxide (20). The mitochondria-produced 
hydrogen peroxide can activate both $\mathrm{Kv}$ channels $(21,22)$ and guanylate cyclase (23), thereby causing pulmonary vasodilatation. By controlling both vascular tone and apoptosis (24), mitochondria are potentially important in the etiology and therapy of vascular disease, but their role in PAH is not known.

Here we investigated a potential role for survivin in PAH. We found that survivin is expressed in remodeled resistance PAs, but not normal PAs, from PAH patients and rats with monocrotalineinduced PAH (MCT-PAH), a widely used model of PAH. Inhaled adenoviral gene therapy with survivin-M reverses MCT-PAH. The therapeutic effect of inhibition of survivin is achieved by induction of mitochondria-dependent apoptosis in PASMCs. Interestingly, this is also associated with activation of $\mathrm{Kv}$ channels.

\section{Results}

Survivin is expressed in the PAs from patients with PAH but not in the PAs from patients without $P A H$. We performed immunohistochemistry in lungs from 10 patients: 6 with PAH, 1 with pulmonary hypertension due to thromboembolic disease, and 3 without pulmonary hypertension (Figure 1 and Table 1). Survivin was expressed in the media and neointima of remodeled resistance PAs in all PAH patients studied (Figure 1A). In contrast, survivin was absent in the PAs from 3 patients without pulmonary hypertension and in the remodeled PAs from a patient with secondary pulmonary hypertension due to thromboembolic disease (Figure 1B). Survivin was expressed in most of the small resistance PAs and often in medium-sized PAs. In the majority of the PAs studied, there was colocalization of survivin with smooth muscle actin, as shown by double staining of the slides and multiphoton laser confocal microscopy. In several small PAs, there was also colocalization of survivin with $\mathrm{WWF}$, which suggests that survivin was also expressed in cells with endothelial features in the obliterated lumen of the small remodeled PAs. At times, cells expressing survivin were found among cells not expressing smooth muscle actin, external to the media; these cells were likely fibroblasts (patients 4 and 5; Figure 1 and Table 1).

Survivin was expressed in the PAs from PAH but not normal rats, and its expression temporally paralleled the rise in PA pressure. Survivin was heavily expressed in the media of rats with MCT-PAH, a widely accepted model of PAH. Similarly to the human tissue, survivin was not expressed in the PAs of normal rats (Figure 2A). Heavy survivin expression was seen in rats with severe PAH (21 days after MCT injection). In contrast, light expression of survivin was seen in partially remodeled PAs, and no expression was seen in non-remodeled PAs from rats with mild early PAH (11 days after MCT) (Figure 2A); this suggests that the expression of survivin is positively associated with the progression and severity of PA remodeling.

To further study the time course of survivin expression relative to the progression of $\mathrm{PAH}$, rats were sacrificed at different time points after the s.c. injection of MCT. The rats underwent echocardiography studies immediately prior to sacrifice, in which we measured PA acceleration time (PAAT), a Doppler parameter that has been shown to correlate well with PA pressure in both humans and rats (as the PA pressure rises, PAAT shortens) $(25,26)$. In parallel experiments, we monitored the PA pressure continuously after MCT injection in freely moving rats with telemetry catheters placed in the PA $(26,27)$. The increase in the PA mRNA for survivin paralleled the increase in PA pressure. Survivin expression peaked at 10 days after MCT injection, and PA pressure started to increase shortly after that time, i.e., approximately 12 days (Figure $2 \mathrm{~B}$ ). Immunoblots of pooled PAs from 3 rats also show that survivin protein expression was increased 10 days after MCT. The timing of the peak of survivin expression at day 10 after MCT is in agreement with the timing of survivin expression in the carotid injury model (18). In contrast, in this early stage of PAH, expression of bcl-2, an antiapoptotic mediator that is upregulated in patients with late/established PAH (7), was not altered. Expression of $\mathrm{Kv} 1.5$, a $\mathrm{K}^{+}$channel downregulated in both animal $(8,9,26)$ and human PAH (28), decreased in parallel to the increase in survivin and preceded the rise in PA pressure (Figure 2B).

Survivin regulates mitochondria-dependent apoptosis and $\mathrm{K}^{+}$current in PASMCs. To study the effect of survivin on PASMCs in vitro, we established a model where primary cultures of PASMCs, isolated from resistance rat PAs (Figure 3A), were exposed to $10 \%$ FBS (a condition that is known to induce survivin expression in arterial SMCs [ref. 18] and promote proliferation) or 0.1\% FBS (a "starvation" condition that does not induce survivin expression [ref. 18] and promotes apoptosis). We infected the PASMCs with replication-deficient type 5 adenoviruses encoding both GFP and WT survivin (Ad-GFP-S) or both GFP and survivin-M (Ad-GFP$\mathrm{S}-\mathrm{M})$; the expression of both the GFP and the survivin genes was driven by a CMV promoter (18). At a dose of $5 \times 10^{9}$ PFUs (100 $\mu \mathrm{l}$ in 60 -mm plates) we achieved infection rates greater than $80 \%$ (Figure 3B). Transfer of WT survivin with Ad-GFP-S in "starved" PASMCs (0.1\% FBS) suppressed apoptosis (measured by TUNEL and DAPI staining) and promoted cell proliferation (measured by expression of the proliferating cell nuclear antigen, PCNA). In contrast, gene transfer of the survivin mutant with Ad-GFP-S-M in PASMCs exposed to $10 \%$ FBS induced apoptosis and decreased PCNA expression (Figure 3, C and D).

We then measured mitochondrial membrane potential using tetramethylrhodamine methyl-ester (TMRM) in PASMCs (26) infected with Ad-GFP-S-M versus Ad-GFP-S. Infection with Ad-GFP-S-M caused significant mitochondrial depolarization (decreased TMRM red fluorescence), compared with noninfected PASMCs from the same plate (Figure 4A). In contrast, gene transfer of WT survivin with Ad-GFP-S caused mitochondrial hyperpolarization, an apoptosis-resistance state. That mitochon-

\begin{tabular}{|c|c|c|c|c|c|}
\hline Patient & Type & Age/Sex & Severity & Epoprostanol & Material \\
\hline 1 & iPAH & $31 \mathrm{M}$ & Severe & + & Transplant \\
\hline 2 & iPAH & $52 \mathrm{M}$ & Severe & + & Transplant \\
\hline 3 & PAH-VSD & $32 \mathrm{~F}$ & Severe & - & Wedge biopsy \\
\hline 4 & iPAH & $48 \mathrm{~F}$ & Severe & - & Transplant \\
\hline 5 & PAH-ASD & $46 \mathrm{~F}$ & Moderate & - & Wedge biopsy \\
\hline 6 & iPAH & $33 \mathrm{~F}$ & Severe & - & Transplant \\
\hline 7 & 2-PHTA & $38 \mathrm{M}$ & Severe & - & Wedge biopsy \\
\hline 8 & Normal & $26 \mathrm{M}$ & & & Donor \\
\hline 9 & Normal & $38 \mathrm{~F}$ & & & Donor \\
\hline 10 & Normal & $42 \mathrm{M}$ & & & Lobectomy ${ }^{B}$ \\
\hline
\end{tabular}

Patient numbers correspond to those in Figure 1, A and B. ASecondary $\mathrm{PAH}$ due to thromboembolic disease. BBenign tumor of the lung. $\mathrm{PAH}$, idiopathic PAH; PAH-VSD, PAH due to ventricular septal defect;

PAH-ASD, PAH associated with atrial septal defect. 


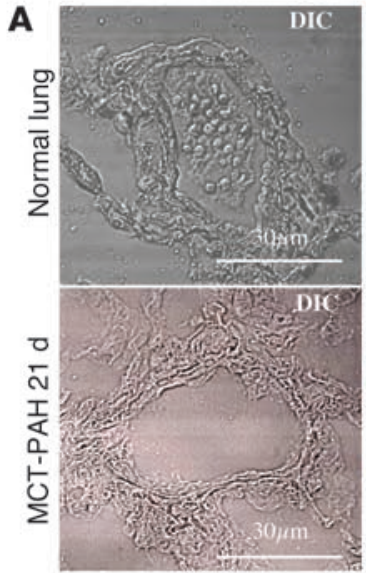

S

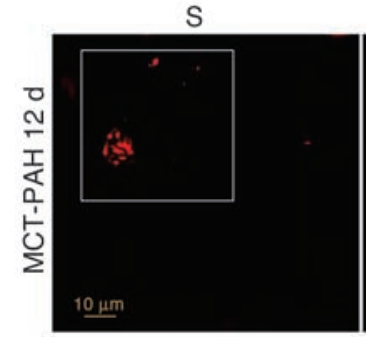

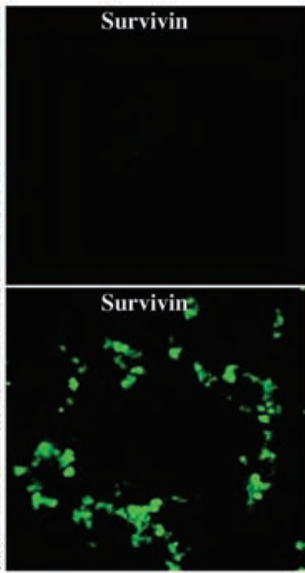

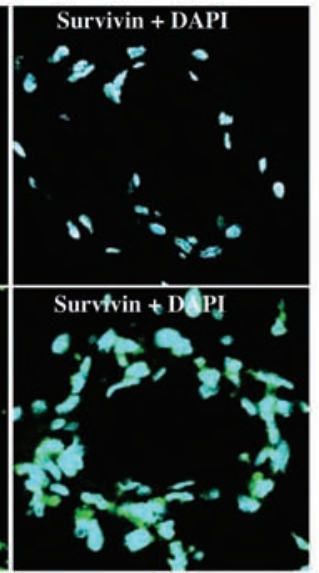

SMA

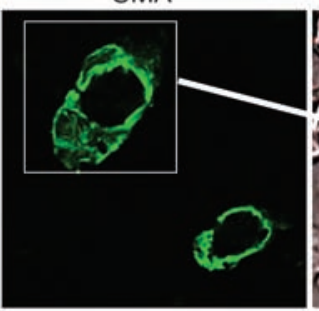

S+SMA+DAPI

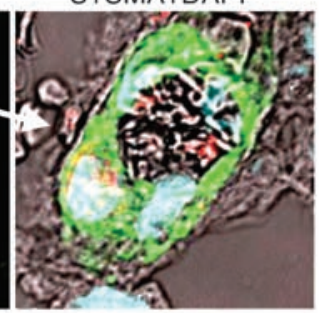

B
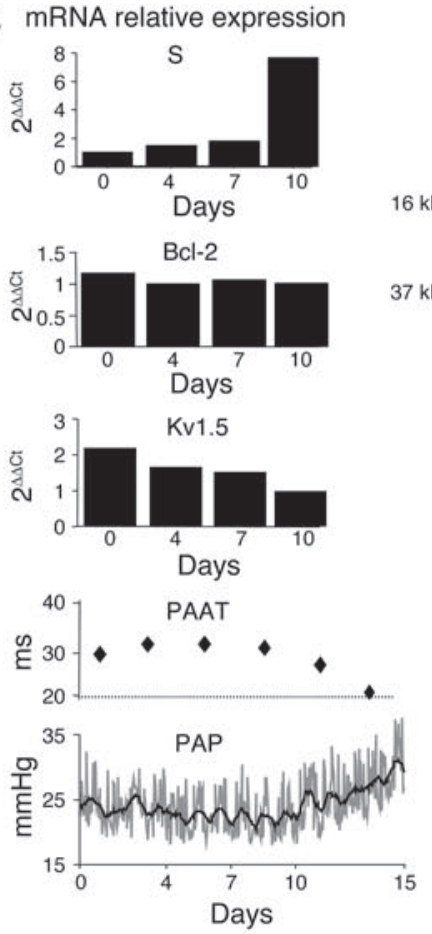

Figure 2

Survivin is expressed in the PAs of rats with MCT-PAH, and its expression parallels the rise in PA pressure. (A) Survivin is expressed in the media of resistance PAs from rats with MCT-PAH but not in PAs from control rats. Note the heavy expression of survivin in severe MCT-PAH (21 days after MCT), compared with mild MCT-PAH (12 days after MCT). In the latter, note that a smooth muscle actin-positive PASMC is heavily expressing survivin in the media of a small PH that shows early medial hypertrophy, whereas no survivin is expressed in another PA from the same field that does not show evidence of medial hypertrophy. Magnification in S+SMA+DAPI, $\times 75$. (B) Survivin expression, measured by quantitative RT-PCR and immunoblots, increases 10 days after MCT injection, prior to the increase of PA pressure as measured by in vivo telemetry (mean PA pressure [PAP] shown) and by echocardiography (PAAT). Kv1.5 expression is decreasing in parallel with survivin, before the rise in pressure, while bcl-2 expression is unchanged.

dria-dependent apoptosis was induced in these PASMCs was also shown by the activation of caspases using immunoblots and cleavage assays; infection with Ad-GFP-S-M, but not with Ad-GFP-S, activated both caspase- 9 and caspase-3 (Figure 4A).

Further exploring the mechanism of mitochondria-dependent apoptosis in our model, we performed fluorescence immunocytochemistry to determine whether cytochrome $c$ leaks into the cytoplasm after the mitochondria depolarization. In all PASMCs infected with Ad-GFP-S-M, the cytochrome $c$ staining showed a diffuse, homogeneous pattern, suggesting leakage to the cytoplasm. In contrast, infection with Ad-GFP-S did not cause cytochrome $c$ release, as shown by the punctate, mitochondrial staining pattern (Figure 4B). Infection with Ad-GFP did not alter the mitochondrial membrane potential and did not cause efflux of cytochrome $c$. We also studied another important mediator of mitochondria-dependent apoptosis, apoptosis-inducing factor (AIF), which causes caspase-independent apoptosis via its nuclease activity in the nucleus (29). In the PASMCs infected with Ad-GFP$\mathrm{S}-\mathrm{M}$, but not in those infected with Ad-GFP-S, AIF was translocated into the nucleus (Figure 4C).

Cytochrome $c$ not only activates caspases but has recently been shown to activate $\mathrm{Kv}$ channels in PASMCs, before inducing apoptosis (30). Since intracellular $\mathrm{K}^{+}$tonically inhibits caspases, the decrease in intracellular $\mathrm{K}^{+}$that follows $\mathrm{Kv}$ channel activation further promotes apoptosis (14). Concordant with the mito- chondrial depolarization and cytochrome $c$ release, the Ad-GFPS-M-infected PASMCs had a significant increase in PASMC $\mathrm{K}^{+}$ current; conversely, Ad-GFP-S-infected PASMCs had a decrease in $\mathrm{K}^{+}$current, compared with noninfected cells, as determined by GFP fluorescence (Figure 5A). The efflux of $\mathrm{K}^{+}$from the cells that follows the $\mathrm{K}^{+}$channel opening contributes to the osmotic shrinkage of cells, an early marker of apoptosis. As shown by the measurement of PASMC capacitance, the "proapoptotic" Ad-GFPS-M-infected PASMCs were smaller than the noninfected controls (Figure 5A). Ad-GFP-S-M did not alter the $\mathrm{K}^{+}$current in "serumstarved" PASMCs; this was expected, since the dominant-negative construct would have no effect in a state in which endogenous survivin is absent. On the other hand, Ad-GFP-S further decreased $\mathrm{K}^{+}$current in PASMCs exposed to $10 \%$ FBS.

Survivin inhibition in vivo improves pulmonary hemodynamics and vascular remodeling and prolongs survival in established MCT-PAH. We delivered the survivin mutant selectively to resistance PAs in the lungs of MCT-PAH rats 12-13 days after injection, when endogenous expression of survivin peaks (Figure $2 \mathrm{~B}$ ), and studied them 2 weeks later. We nebulized the Ad-GFP-S-M versus a virus carrying GFP only (Ad-GFP) and delivered them intratracheally. We have previously shown that this route of delivery results in transgene expression that is restricted to the lung and that persists for at least 2 weeks (9). Diffuse GFP immunofluorescence throughout the vascular wall in medium-sized and small PAs con- 
A

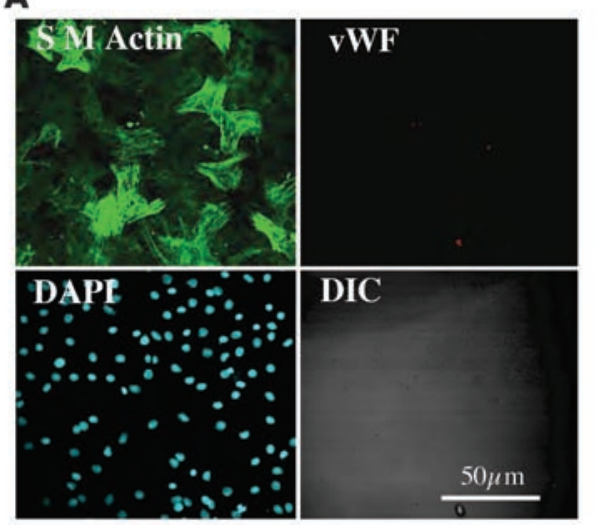

B
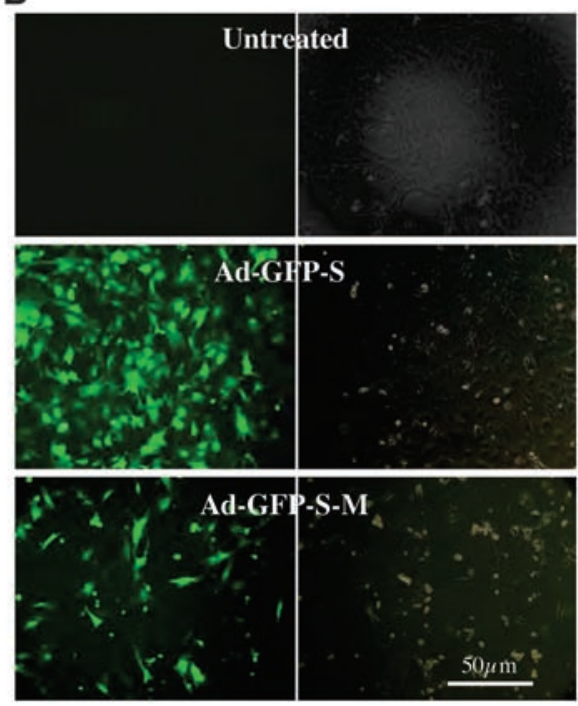

C

GFP

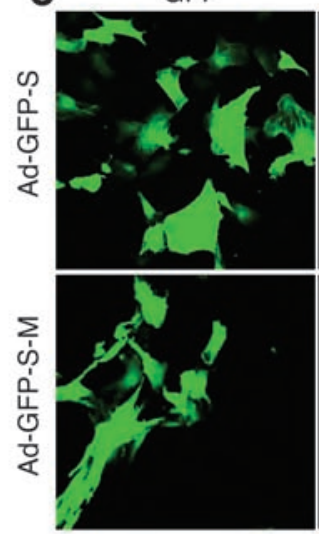

GFP
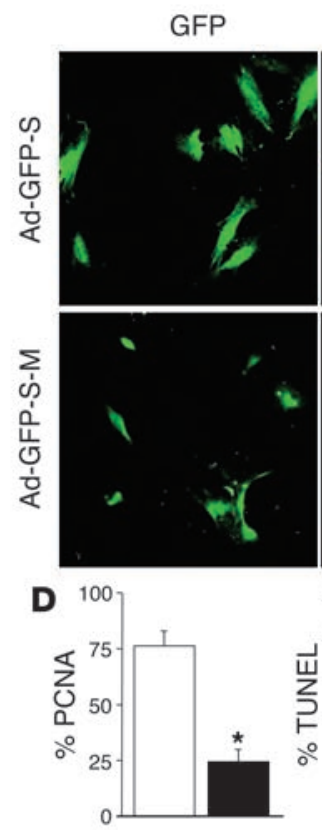

TUNEL

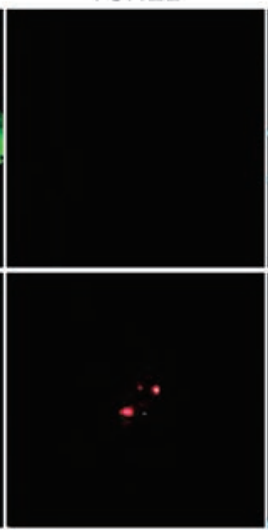

PCNA
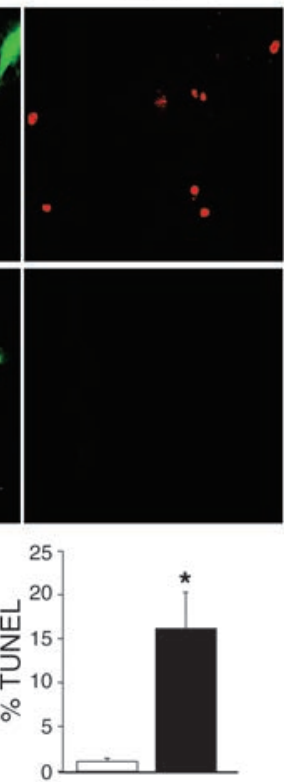

All
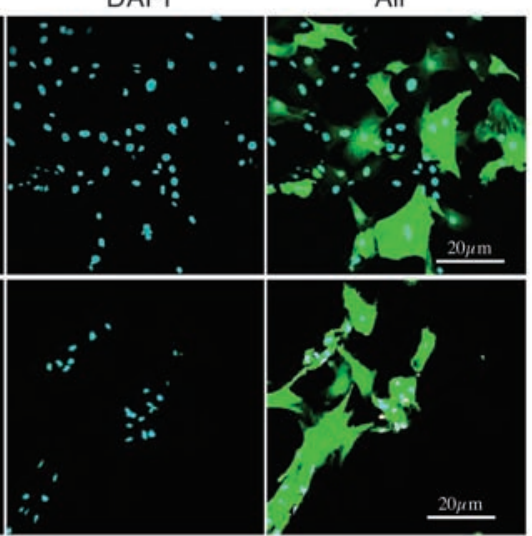

DAPI

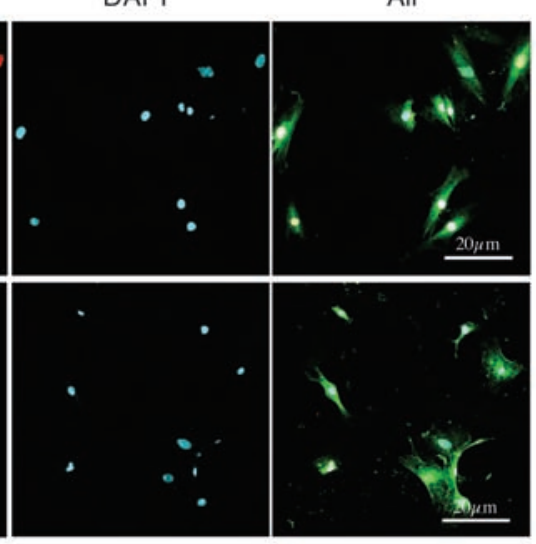

$\square$ Ad-GFP-S

Ad-GFP-S-M

Figure 3

Efficient adenoviral delivery of phosphorylation-deficient survivin (Ad-GFP-S-M) to PASMCs in vitro reduces proliferation and increases apoptosis. (A) Primary culture of rat PASMCs stains positive for smooth muscle actin but not vWF, indicating no contamination with endothelial cells. (B) Infection of PASMCs with adenoviruses encoding GFP and either WT survivin (Ad-GFP-S) or survivin mutant (Ad-GFP-S-M) was highly efficient, as evidenced by GFP reporter (green fluorescence in the left panels and differential interference contrast [DIC] in the right panels). Note the reduced cellularity of the plate infected with Ad-GFP-S-M, compared with Ad-GFP-S. (C) Cells infected with Ad-GFP-S-M (grown in 10\% FBS) show increased TUNEL-positive nuclei and reduced PCNA-positive nuclei, suggesting that they undergo apoptosis and not proliferation. In contrast, cells infected with Ad-GFP-S (grown in $0.1 \%$ FBS) show no apoptosis and increased rates of PCNA expression. (D) Mean data for TUNEL- and PCNA-positive nuclei, 48 hours after infection with Ad-GFP-S-M versus Ad-GFP-S; 5 fields studied in each plate, 20 plates per group. ${ }^{*} P<0.01$.

firmed the effective gene transfer (Figure 5B). Since the small resistance PAs control most of PVR, we quantified the expression of GFP in the microvasculature using laser-capture microdissection and quantitative RT-PCR (Figure 5B). We found that gene delivery was effective particularly in the very small (less than $50 \mu \mathrm{M}$ ) PAs. This is expected, since these PAs are surrounded by alveoli and are directly accessible to the viruses.

In order to study the selectivity and safety of our gene delivery method, we measured the expression of GFP and survivin in treated and untreated rats. We showed that the mRNA levels of survivin in normal and MCT-PAH rats were extremely low in the liver, heart, kidney, and muscle but were unexpectedly high in the spleen. The levels of endogenous survivin were also extremely low in the healthy lung but significantly increased in the treated rats. The significance of the high levels of survivin in the spleen, an organ critical for the immune response, is unclear at this point. If our Ad-GFP-S-M virus were to reach the spleen, the inhibition of the endogenous survivin might have deleterious effects. However, our inhaled gene therapy adds another level of selectivity to our approach. Indeed, in the treated rats, GFP was not detected at all in all organs measured, including the spleen, except the lung (Figure 5C). Further supporting the safety and the selectivity of our approach is the blood work listed in Table 2, showing lack of any hematologic, liver, or renal toxicity during the study period. 
A

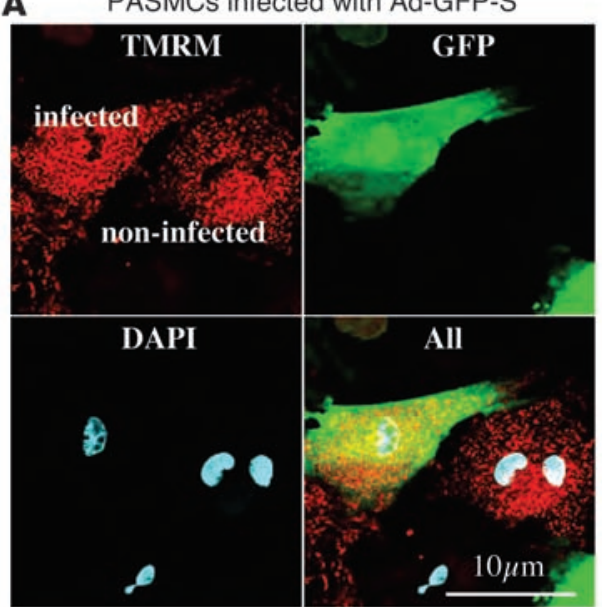

B
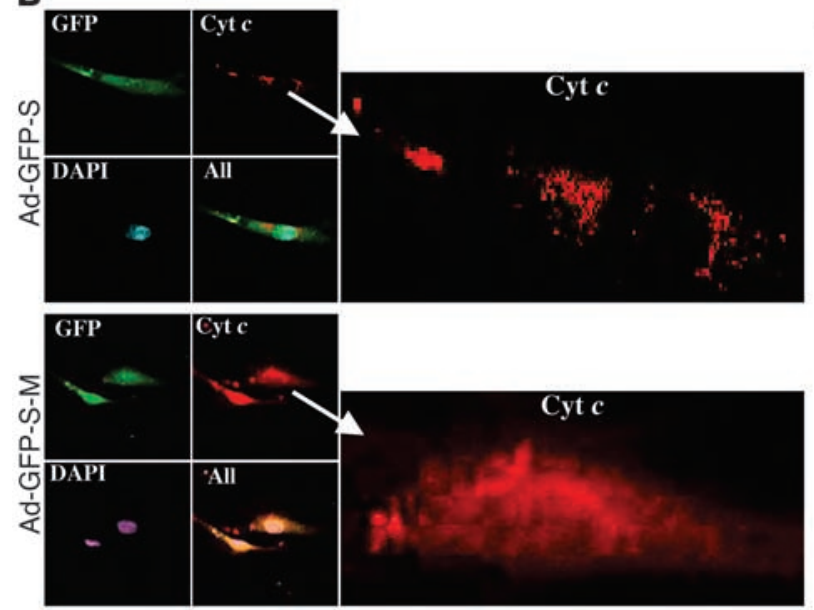

PASMCs infected with Ad-GFP-S-M

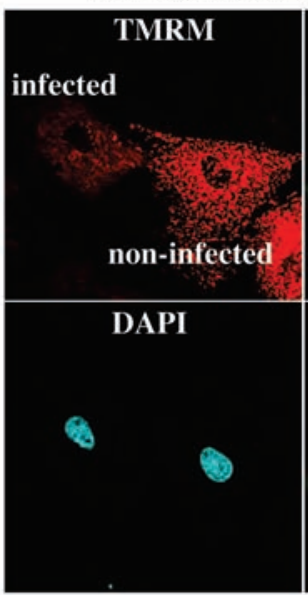

TMRM red fluorescence (FU)
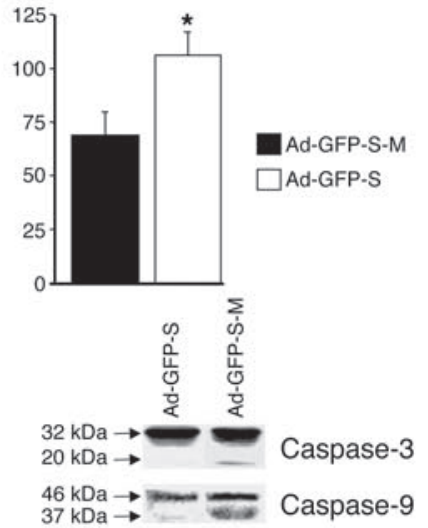

$10 \mu \mathrm{m}$
C
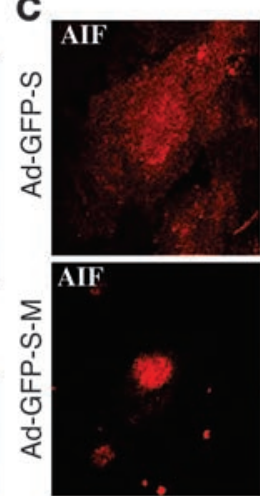
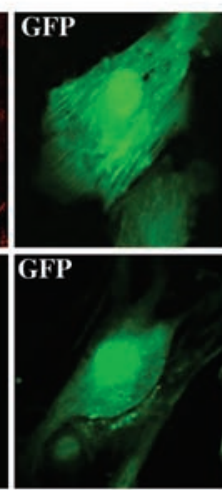

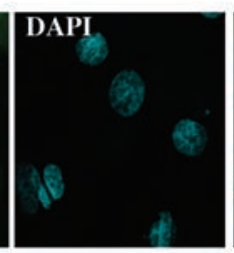

DAPI

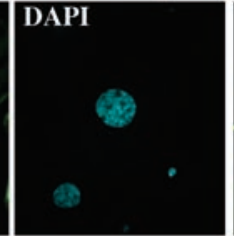

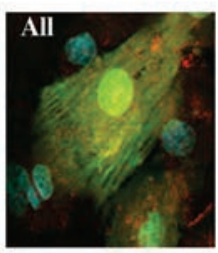

All

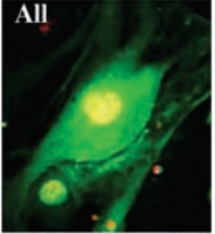

\section{Figure 4}

Ad-GFP-S-M infection induces PASMC mitochondria-dependent apoptosis. (A) PASMCs effectively infected with Ad-GFP-S (green) show slight hyperpolarization of mitochondrial membrane potential (increased TMRM fluorescence) compared with noninfected cells. In contrast, mitochondria of Ad-GFP-S-M-infected cells (but not neighboring noninfected cells) are less red, indicating depolarized mitochondria. Mean data are shown on the right (arbitrary fluorescence units [FU], means from 15 plates per group; ${ }^{*} P<0.01$ ). Immunoblots show that, in contrast to expression of WT survivin, expression of survivin mutant in PASMCs grown in 10\% FBS induces activation of caspase-9 and caspase-3. (B) PASMCs infected with Ad-GFP-S demonstrate sequestered cytochrome $c$ within mitochondria, as shown by the punctate pattern of staining. In contrast, mitochondria of Ad-GFP-S-M-infected cells show cytochrome $c$-positive staining diffusely throughout the cell, indicating leakage of cytochrome $c$ from mitochondria into the cytoplasm. Magnification: left and middle panels, $\times 75$; right panels, $\times 125$. (C) In contrast to infection with Ad-GFP-S, infection with Ad-GFP-S-M induces translocation of the mitochondria-based apoptosis-inducing factor (AIF) in the nucleus, where it initiates caspase-independent apoptosis. Magnification, $\times 100$.

Gene therapy with Ad-GFP-S-M, but not with Ad-GFP, caused an approximately 50\% reduction in PVR index (PVRi), essentially normalizing the PVRi in the treated rats $(P$ value in treated versus untreated rats is not significant). This was accomplished without the alteration of systemic vascular resistance, as expected with selective aerosol gene delivery to the lungs (Figure 6A and Table 2).

In order to obtain a more physiologic assessment of the pulmonary circulation, we also used Doppler echocardiography in intact rats (before the invasive studies) and showed that the Ad-GFP-S-M-treated rats had improved PAAT, in comparison with the untreated and the Ad-GFP-treated rats (Figure 6B). Our PAAT values correlate strongly with our invasively measured PVRi (the higher the PVRi the lower the PAAT; $r^{2}=0.86, P<0.0001, n=57$ rats, i.e., all the rats from our reversal protocol).
In order to determine whether our gene therapy reduced the vascular remodeling in MCT-PAH, we studied PA medial hypertrophy. The Ad-GFP-S-M-treated, but not the Ad-GFP-treated, rats showed a significant reduction in the percentage medial thickness in small and medium-sized PAs (Figure 6C). In contrast, our gene therapy did not alter the vessel density; the number of arteries per low-power field (magnification, $\times 10$ ) was not statistically different among the control, the MCT-PAH, and the Ad-GFP-S-M-treated arteries.

Furthermore, RV hypertrophy (RVH) improved with the Ad-GFP-S-M, but not with the Ad-GFP, gene therapy. The regression of RVH was shown both macroscopically, by measurement of the $\mathrm{RV} /(\mathrm{LV}+$ septum) ratio, and echocardiographically, by measurement of the RV free wall thickness in vivo (Table 2). 
A
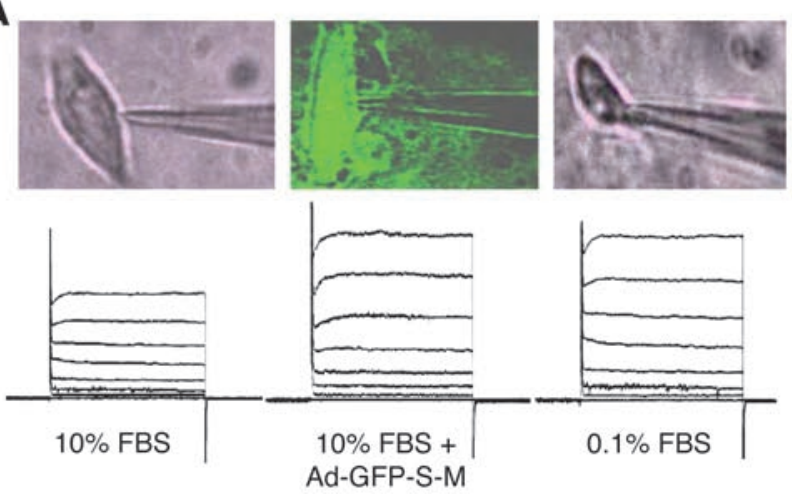

Ad-GFP-S-M
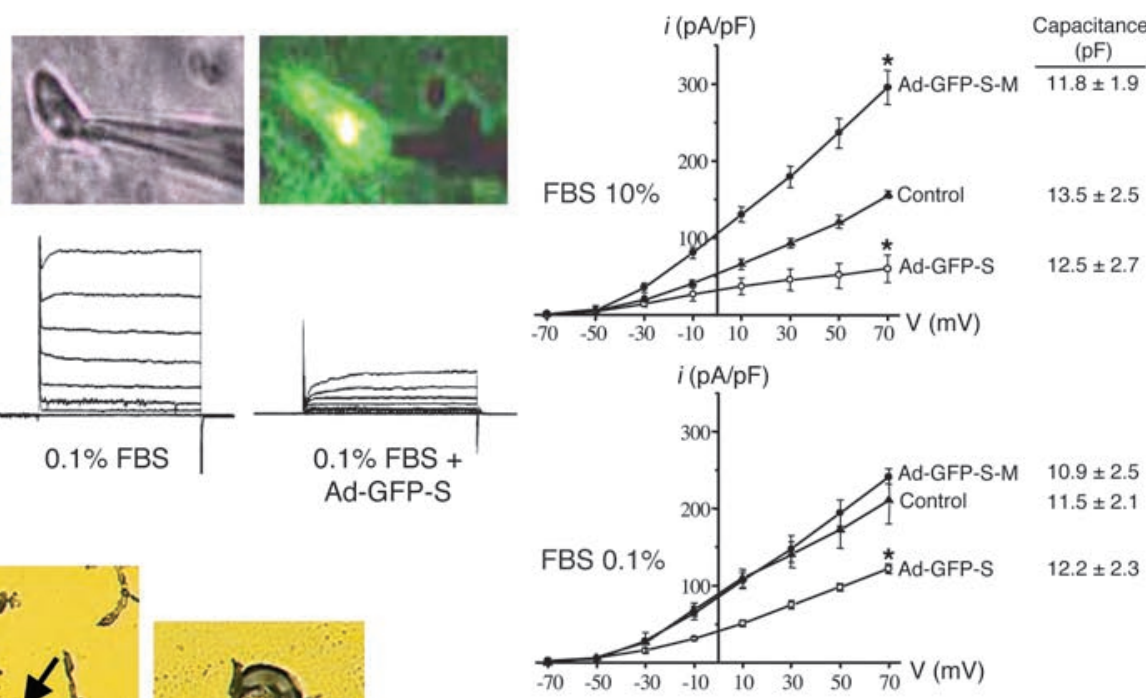

B
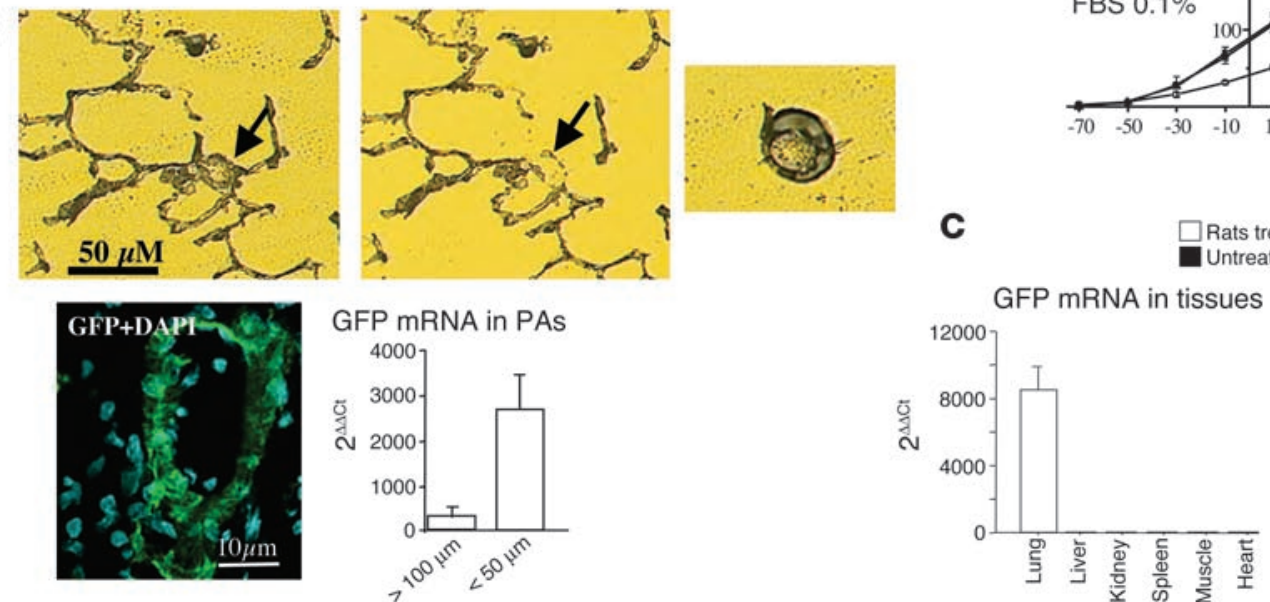

treated with Ad-GFP-S-M

reated, normal rats
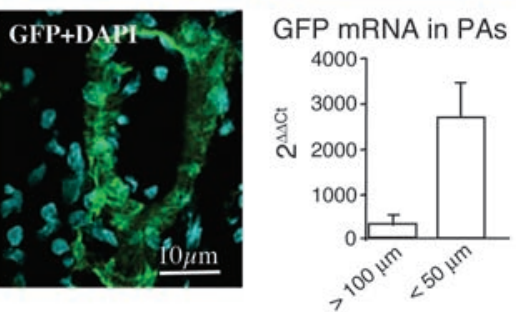

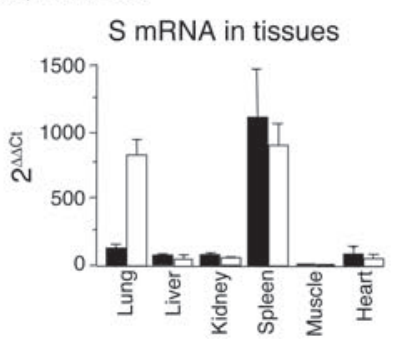

Figure 5

Selective expression of survivin mutant in resistance PAs causes an increase in PASMC outward $\mathrm{K}^{+}$current. (A) In FBS-rich conditions (10\% FBS in the medium, a condition known to increase endogenous survivin), infection with Ad-GFP-S-M causes augmentation of $\mathrm{K}^{+}$currents and decreased capacitance $(\mathrm{Cm}$, a measure of cell size), consistent with apoptosis; the opposite is seen with Ad-GFP-S infection. In contrast, in serum-deprived conditions ( $0.1 \% \mathrm{FBS})$, infection with Ad-GFP-S causes a decrease in $\mathrm{K}^{+}$currents, consistent with apoptosis resistance. Since in these conditions endogenous survivin is absent, infection with Ad-GFP-S-M has no effect on $\mathrm{K}^{+}$current. Cells carrying the transgenes were selected by the green fluorescence. Mean data for current density over voltage are shown on the right $\left(n=6\right.$ cells per group; ${ }^{*}<<0.01$ vs. control). (B) Both GFP immunofluorescence microscopy and quantitative RT-PCR of laser-capture-microdissected resistance PAs demonstrate efficient delivery of the transgenes, particularly to the very small (less than $50 \mu \mathrm{m}$ ) resistance PAs (arrows). (C) In our inhaled gene therapy approach, the expression of the transgenes is restricted to the lungs, as shown by the expression of GFP, measured by quantitative RT-PCR. The expression of endogenous survivin in nontreated rats is minimal in all organs studied, except the spleen. Our WT-survivin primer also detects the survivin mutant, as shown by the increased lung signal in the treated rats. Data from 5 rats per group are shown.

Two-dimensional echocardiography also showed that the shift in the interventricular septum seen in the MCT-PAH rats was normalized in the Ad-GFP-S-M-treated rats, confirming effective decrease in PA pressures (Figure 6B). The significant improvement in the pulmonary hemodynamics and RVH may explain the $25 \%$ reduction in mortality (Figure $6 \mathrm{D}$ ), similar to the result of another experimental therapy inducing mitochondria-dependent apoptosis in the same model (26).

It has been suggested that in the MCT model of PAH, endothelial loss occurs early and leads to loss of microvessels and PAH; suppressing apoptosis with cell-mediated gene therapy with angiopoietin-1 leads to prevention of MCT-PAH (31). In addition to not finding any difference in vessel density, here and in our recent work (26) we could find no evidence of increased apoptosis early in MCT-PAH, although this possibility cannot be excluded at this point. Nevertheless, theoretically, our pro- apoptotic strategy could exacerbate endothelial cell loss early on and worsen PAH. Therefore, we delivered the Ad-GFP-S-M and Ad-GFP on day 1 of the MCT injection, and we studied the rats 3 weeks later ( $n=6$ rats per group). The severity of PAH was similar to that seen with the reversal protocol, but there were no differences in the pulmonary and systemic hemodynamics between the Ad-GFP and Ad-GFP-S-M groups $(P<0.8)$. The lack of any effect from our gene therapy on day 1 is in agreement with the lack of significant endogenous survivin expression during the first 10 days after MCT (Figure 2B).

The reversal of vascular remodeling by survivin targeting is caused by induction of apoptosis, suppression of proliferation, and activation of $K v$ channels in PASMCs. The survivin mutant gene transfer increased apoptosis (measured by TUNEL) and decreased PCNA expression in the PA media, in agreement with our in vitro data (Figure 7A). BrdU incorporation in PA media was decreased in Ad-GFP-S-M- 


\section{Table 2}

Hemodynamic and toxicity data of the survivin-targeting gene therapy in established MCT-PAH

$\begin{array}{lcccc} & \text { Control } & \text { MCT } & \text { +Ad-GFP } & \text { +Ad-GFP-S-M } \\ \text { PVRi }(\mathrm{mmHg} \times \min \times \mathrm{g} / \mathrm{ml}) & 35 \pm 6 & 121 \pm 10 & 117 \pm 9 & 57 \pm 3^{\mathrm{A}} \\ \text { SVRi }(\mathrm{mmHg} \times \mathrm{min} \times \mathrm{g} / \mathrm{ml}) & 269 \pm 16 & 282 \pm 8 & 269 \pm 15 & 254 \pm 9 \\ \text { Mean PAP }(\mathrm{mmHg}) & 17 \pm 4 & 40 \pm 3 & 39 \pm 2 & 25 \pm 1^{\mathrm{A}} \\ \text { LVEDP }(\mathrm{mmHg}) & 4.3 \pm 0.3 & 4.7 \pm 0.2 & 4.1 \pm 0.4 & 4.8 \pm 0.2 \\ \text { Systolic BP }(\mathrm{mmHg}) & 91 \pm 1 & 82 \pm 1 & 80 \pm 4 & 92 \pm 2^{\mathrm{B}} \\ \text { Heart rate }(\text { beats/min) } & 260 \pm 18 & 257 \pm 7 & 265 \pm 9 & 245 \pm 7^{\mathrm{A}} \\ \text { Cl (ml/min/g) } & 349 \pm 25 & 294 \pm 8 & 299 \pm 14 & 366 \pm 8^{\mathrm{A}} \\ \text { RV/(LV + septum) } & 0.29 \pm 0.001 & 0.51 \pm 0.03 & 0.51 \pm 0.02 & 0.37 \pm 0.14^{\mathrm{A}} \\ \text { PAAT (ms) } & 31 \pm 1 & 18 \pm 2 & 18 \pm 1 & 26 \pm 1^{\mathrm{A}} \\ \text { RV thickness (mm) } & 0.6 \pm 0.02 & 0.9 \pm 0.05 & 0.9 \pm 0.04 & 0.7 \pm 0.03^{\mathrm{B}} \\ \text { Hgb }(\mathrm{g} / \mathrm{l}) & 150 \pm 2 & 150 \pm 6 & & 148 \pm 2 \\ \text { wbc } & 9 \pm 3 & 12 \pm 3 & & 10 \pm 2 \\ \text { Platelet } & 1,010 \pm 56 & 1,029 \pm 74 & & 1,096 \pm 98 \\ \text { AST (U/l) } & 80 \pm 5 & 70 \pm 9 & & 80 \pm 2 \\ \text { Creatinine }(\mu \mathrm{M} / \mathrm{l}) & 46 \pm 4 & 52 \pm 9 & & 50 \pm 13 \\ & & & & \end{array}$

AP $<0.0001$ vs. Ad-GFP; ${ }^{B} P<0.001$ vs. Ad-GFP. PVRi, pulmonary vascular resistance stolic pressure; $\mathrm{Cl}$, cardiac index; Hgb, hemoglobin; AST, aspartate aminotransferase. index; SVRi, systemic vascular resistance index; PAP, PA pressure; LVEDP, LV end-dia-

endothelial injury selectively in the pulmonary circulation (32), since its active toxic metabolite is secreted by the liver. Endothelial damage results in exposure of PASMCs to circulating growth factors, including PDGF, which is increased early in MCT-PAH, before the rise in PA pressure (33), in agreement with the survivin expression profile (Figure 2B). Indeed, MCT$\mathrm{PAH}$ can be prevented by prophylactic preservation of the endothelium, where the early endothelial cell loss is prevented by gene transfer of the antiapoptotic angiopoietin-1 at the time of MCT injection (31). Delivering the proapoptotic Ad-GFP-S-M on day 1 did not prevent PAH but also did not worsen it compared with Ad-GFP. This was expected, since the lack of endogenous survivin the first week after MCT explains the lack of any effect of the dominantnegative survivin mutant delivered with our replication-deficient adenovirus. This also proves that the beneficial effects of the Ad-GFP-S-M in the reversal protocol were not nonspecific but resulted from the inhibition of endogenous survivin, which is expressed during a specific window in the development of $\mathrm{PAH}$, i.e., 10 days after MCT exposure.

Because PAH patients present late in their clinicompared with Ad-GFP-treated rats, consistent with inhibition of cell proliferation (Figure 7B).

In agreement with our in vitro data, freshly isolated PASMCs from the Ad-GFP-S-M-treated, but not the Ad-GFP-treated, rats had increased $\mathrm{K}^{+}$current, compared with those from the MCT$\mathrm{PAH}$ rats (Figure $7 \mathrm{C}$ ). The current morphology and sensitivity to 4-aminopyridine (a relatively specific Kv channel blocker at $5 \mathrm{mM}$ ) showed that the induced current was conducted by $\mathrm{Kv}$ channels (Figure 7C).

To further support the hypothesis that survivin is directly involved in the pathogenesis of PAH, we delivered WT survivin in normal rats and studied them 2 weeks later ( $n=5$ per group). Inhaled delivery of Ad-GFP-S in healthy rats caused a mild but significant increase in the medial hypertrophy and PA pressure as well as RVH (Figure 8).

\section{Discussion}

Here we show that survivin is etiologically associated with the development of $\mathrm{PAH}$, and we open a new window for the treatment of this devastating disease. Survivin is expressed in established human and experimental PAH, but not in normal PAs. Its expression parallels the rise of PA pressure, although it is not known whether this is true in human PAH. Inhibition of endogenous survivin significantly improves PVRi, PA medial hypertrophy, and RVH, prolonging survival by $25 \%$; in contrast, delivery of WT survivin increases PA pressure, medial hypertrophy, and RVH. We provide direct evidence that both in vitro and in vivo survivin targeting induces PASMC mitochondria-dependent apoptosis and is associated with activation of Kv channels. Our inhaled gene therapy approach is highly selective, with transgene expression restricted in the pulmonary circulation; the reversal of PAH is achieved without any hematologic, liver, or renal toxicity.

Survivin is selectively expressed in cancer because of oncogenic transformation (16). In vascular SMCs in vitro, survivin expression is increased by exposure to serum growth factors like PDGF (18). MCT is an endothelial toxin, and a single injection results in cal course, reversal approaches are much more clinically relevant than prevention. Although increased endothelial apoptosis might indeed be a very early feature of $\mathrm{PAH}$, the suppressed apoptosis and proliferative remodeling in the media that occur later and persist in established PAH are a more therapeutically relevant abnormality. The modulation of apoptosis and proliferation by our gene therapy is localized primarily in the media and PASMCs, as suggested by our in vitro data and vascular histology, although effects on the endothelium or fibroblasts cannot be excluded. In the severe cases of human PAH, survivin was often expressed in cells expressing vWF, in addition to PASMCs, in the remodeled small PAs (Figure 1A). The exact origin of these cells in the remodeled PA is not clear (native vascular cells "invading" from the adventitia and the media, versus circulating precursor cells as part of a distorted regenerative response). Nevertheless, apoptosis of any proliferating cell obstructing the lumen might be beneficial. However, this remains a speculation, and more experiments are needed to determine the relative role of PASMCs and endothelial cells in our proposed model.

The exact role, location, and timing of apoptosis in PAH remain unknown. In support of our findings that induction of apoptosis in the PA media is beneficial in established PAH, are recent studies showing that serine-elastase inhibitors (34), simvastatin (35), and Rho-kinase inhibitors (36) cause regression of established MCT-PAH by inducing PASMC apoptosis. Furthermore, very recent findings from our group show that activation of mitochondria-dependent apoptosis in the PA media by the metabolic modulator dichloroacetate (an inhibitor of the mitochondria enzyme pyruvate dehydrogenase kinase) also leads to reversal of MCT-PAH and an increase in survival. In a striking similarity with the survivin-targeting gene therapy, treatment with dichloroacetate caused depolarization of mitochondria and activation of $\mathrm{Kv}$ channels, both in vitro and in vivo, and normalized PVR and RVH, without affecting systemic hemodynamics. Taken together, these data suggest that a dysregulation of a mitochondria-Kv channel axis in PAH might be targeted therapeutically with drugs or gene therapies, with similar beneficial effects. 

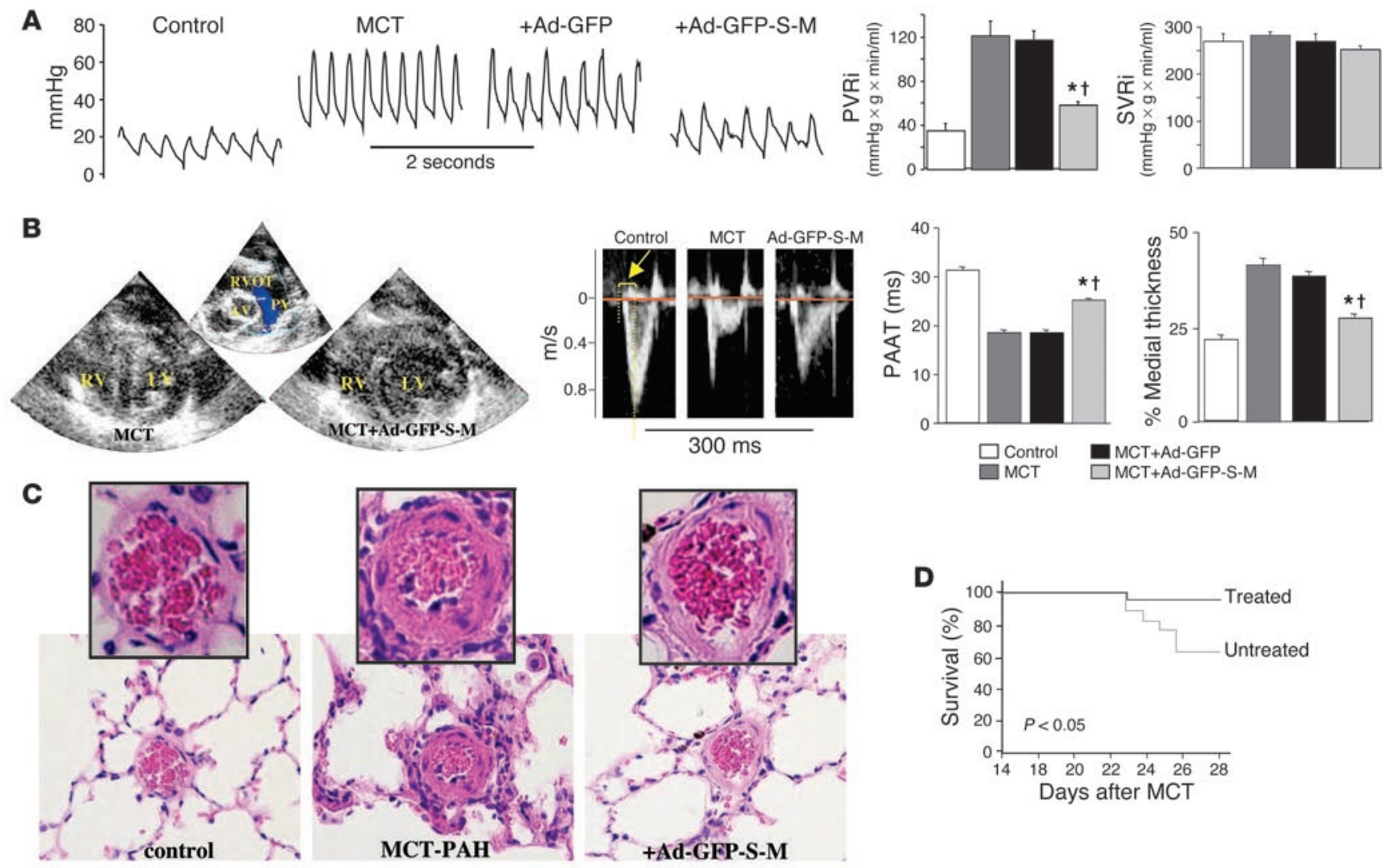

Figure 6

Gene therapy of rat MCT-PAH with Ad-GFP-S-M improves hemodynamics, reduces remodeling of the resistance PAs, and prolongs survival. (A) Representative high-fidelity PA pressure tracings and mean data show that Ad-GFP-S-M, but not Ad-GFP, therapy reduces PA pressure and PVRi, without altering systemic hemodynamics. SVRi, systemic vascular resistance index. (B and C) Ad-GFP-S-M, but not Ad-GFP, reduces RV thickness measured in parasternal short axis (see also Table 2) and preserves the normal round shape of the LV. Similarly, Ad-GFP-S-M reduces PAAT. Resistance PA remodeling, as measured by percent medial thickness, is reduced by treatment with Ad-GFP-S-M. RVOT, RV outflow tract; AV, aortic valve; PV, pulmonary valve. Magnification in C, $\times 40$. (D) Targeting survivin with inhaled gene therapy in MCT-PAH significantly prolongs survival within the study period. ${ }^{*} P<0.05$ vs. MCT; ${ }^{\dagger} P<0.05$ vs. Ad-GFP.

The fact that the pulmonary circulation is selectively diseased in human PAH is a major therapeutic challenge. The majority of drugs targeting the vasculature will, if given systemically, affect the healthy normal circulation as well, thereby limiting efficacy. For example, L-type $\mathrm{Ca}^{++}$channel blockers are useful in a subset of PAH patients (37) but are often not tolerated at useful doses because they cause systemic vasodilatation and hypotension (1). Discovery of factors selectively expressed in the PAs, in addition to novel methods of delivering treatment selectively to the pulmonary circulation (such as inhaled delivery of drugs or genes), is critical. The airway administration of a survivin dominant-negative construct satisfies both requirements to ensure selective targeting of the diseased circulation. This is particularly true since survivin is selectively expressed in the PA media in PAH, but absent in quiescent systemic vascular SMCs (18) and normal PAs (Figures 1 and 2 and Table 1 ).

Our data strengthen the recently proposed view that survivin, in addition to the effects on cell cycle and cell proliferation, also regulates apoptosis $(16,17)$. We show that overexpression of WT survivin hyperpolarizes whereas survivin mutant depolarizes PASMC mitochondria, an initiating event in mitochondria-dependent apoptosis (Figure 4). We also show that this mitochondria depolarization is associated with a leak of cytochrome $c$ and AIF in the cytoplasm. Cytochrome $c$ is a known activator of effector caspases and can also activate PASMC Kv channels (30). Furthermore, depolarized PASMC mitochondria are known to produce more hydrogen peroxide than healthy mitochondria $(20,26,38)$. At low doses (as produced in vivo), hydrogen peroxide is a pulmonary vasodilator and $\mathrm{K}^{+}$channel opener (21-23). The leakage of cytochrome $c$ in the cytoplasm and the increased hydrogen peroxide production might explain the increase in the Kv channel current that we observed in PASMCs both in vitro and vivo (Figure $5 \mathrm{~A}$ and Figure $7 \mathrm{C}$ ). This activation of $\mathrm{K}^{+}$currents that results from survivin targeting is likely to be important in $\mathrm{PAH}$, which is associated with selective inhibition of $\mathrm{Kv}$ channels and is improved by exogenous delivery of Kv1.5 (9). The Kv channel activation associated with survivin targeting is unexplored in cancer, where there is also recent evidence that overexpression of $\mathrm{K}^{+}$channels can induce apoptosis and decrease proliferation in malignant cells (39).

Since survivin expression allows the cells to enter a proliferative phase, inhibition of survivin will result in selective apoptosis of the proliferating PASMC compartment in the vascular wall, sparing the quiescent cells. In other words, it is the subset of PASMCs that express PCNA or take up BrdU that will become apoptotic in the 
A
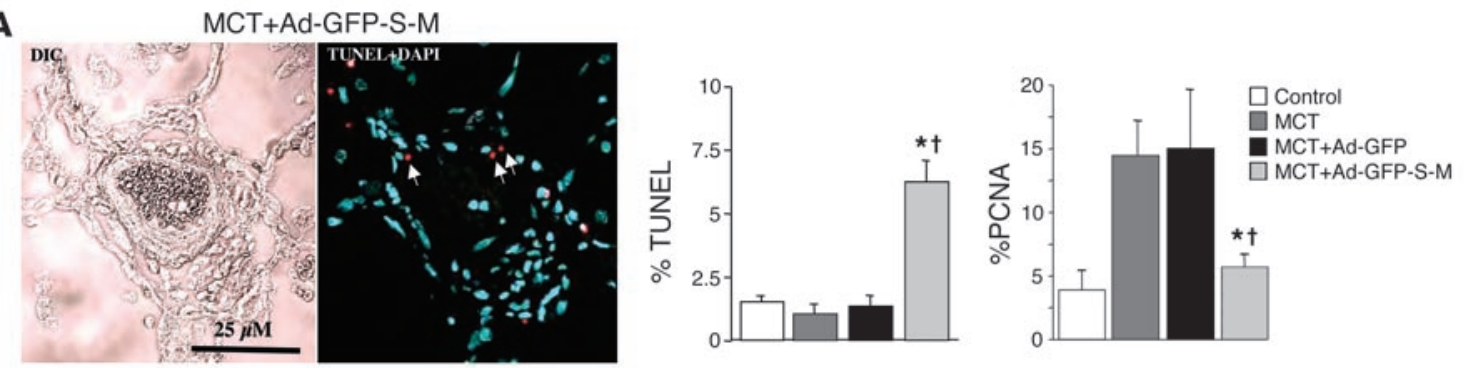

B
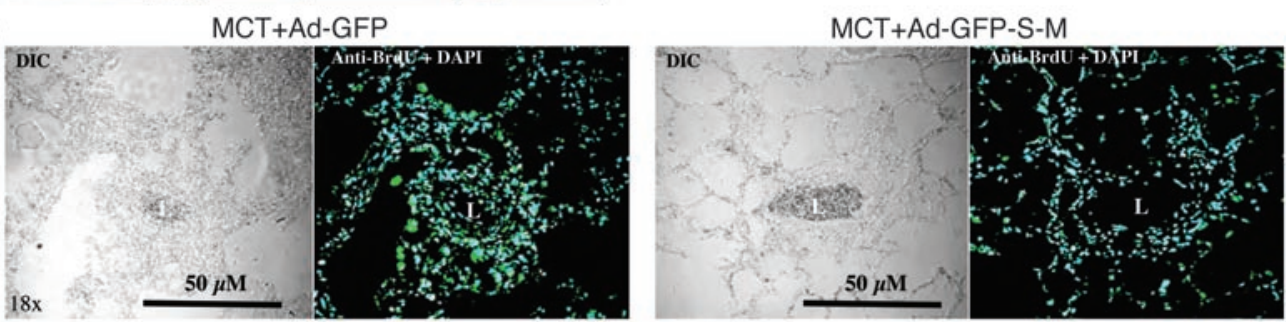

C
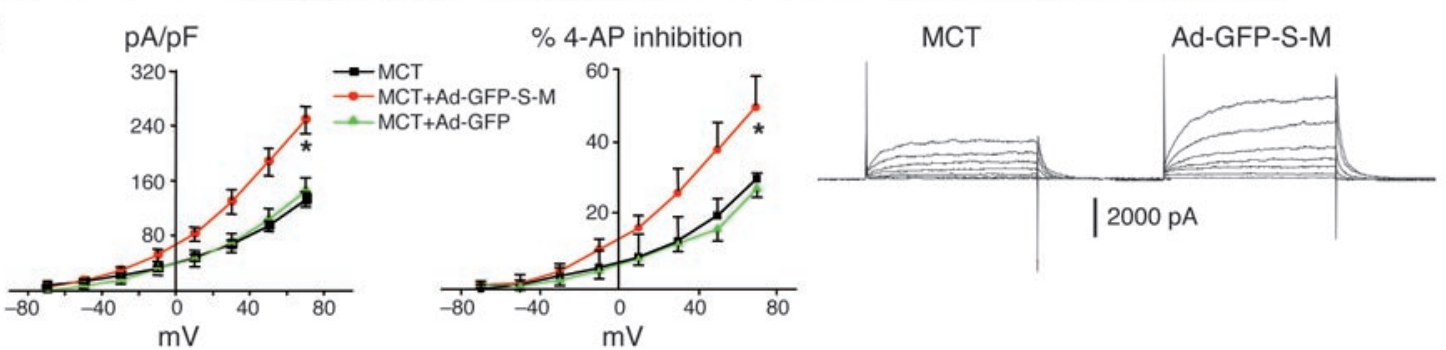

Figure 7

Ad-GFP-S-M augments apoptosis and Kv current and reduces proliferation within resistance PAs in vivo. (A) The number of TUNEL-positive nuclei (arrows) is increased by Ad-GFP-S-M treatment, while the number of PCNA-positive nuclei is reduced, compared with those in the Ad-GFP-treated and nontreated MCT-PAH rats. ${ }^{*} P<0.05$ vs. MCT; ${ }^{\dagger} P<0.05$ vs. Ad-GFP. (B) Reduced BrdU staining (green) in resistance PAs of rats treated with Ad-GFP-S-M, compared with Ad-GFP-S (a representative image from 5 rats per group is shown). (C) Freshly isolated PASMCs from rats treated with Ad-GFP-S-M have increased $\mathrm{K}^{+}$currents, in agreement with our in vitro data (Figure $5 \mathrm{~A}$ ). The sensitivity to 4-aminopyridine (4-AP; $5 \mathrm{mM}$ ) and current morphology suggest that the induced current is voltage-gated (Kv). ${ }^{*}<0.05$ vs. Ad-GFP.

survivin mutant-treated animals. This explains our observation that, both in vivo and in vitro, the survivin mutant-treated vessels had decreased PCNA and BrdU staining (Figures 3 and 7). That survivin targeting selectively causes apoptosis of the proliferating PASMCs is in agreement with the kinetics of apoptosis in cell cycle-synchronized cultures of tumor cells exposed to the same survivin mutant (40).

We offer the following model for the role of survivin in the vascular biology of PAH (Figure 9): Survivin mutations, similar to those observed in oncogenic transformation (41), might occur in some patients with $\mathrm{PAH}$, resulting in spontaneous survivin expression, although this is entirely speculative. Voelkel, Tuder, and colleagues have exposed fascinating similarities between cancer and PAH, by showing that the proliferating cells in the neointimal plexogenic $\mathrm{PAH}$ lesions are monoclonal (42). Alternatively, survivin expression may be induced following endothelial damage, which is widely recognized as a critical early event in the pathogenesis of PAH (31, 43). Endothelial damage would allow direct exposure of PASMCs to circulating growth factors that induce survivin expression. In addition to facilitating the PASMC transition to a proliferative state, survivin would inhibit apoptosis by hyperpolarizing mitochondria. This would result in less cytochrome $c$ and $\mathrm{H}_{2} \mathrm{O}_{2}$ in the cytoplasm, decreasing the tonic activation of $\mathrm{Kv}$ channels. The suppression of $\mathrm{Kv}$ channel activity would cause an increase in the intracellular $\mathrm{K}^{+}$, further suppressing apoptosis, and, by depolarizing the cell membrane, would increase the opening of the voltage-gated $\mathrm{Ca}^{++}$channels; this would increase the influx of $\mathrm{Ca}^{++}$, causing vasoconstriction and amplification of proliferative signal pathways.

The proposed causative role of survivin in PAH and the lack of its expression in quiescent cells in the PA wall and the systemic vasculature make this pathway attractive for future $\mathrm{PAH}$ therapies. However, PAH is a multifactorial disease, and activation of the survivin axis might be only 1 of several abnormalities that contribute to the development of PAH in a given patient. Nevertheless, the lungselective inhaled gene therapy approach, the lack of systemic toxicity, and its effectiveness in prolonging survival make survivin targeting an attractive candidate therapy for human PAH. Our proposed survivin-mitochondria-Kv channel axis merits further assessment not only in vascular biology but in cancer biology as well.

\section{Methods}

Experimental protocol. All experiments were conducted with the approval of the University of Alberta Animal Policy and Welfare Committee. Age- and weight-matched male Sprague-Dawley rats (250-300 g) were used.

In the reversal protocol, rats were randomized to: control (sham saline injection, $n=7), 60 \mathrm{mg} / \mathrm{kg} \mathrm{MCT} \mathrm{s.c.}(n=20$; Sigma-Aldrich), MCT + Ad-GFP $(n=10)$, and MCT+Ad-GFP-S-M $(n=20)$. PFUs $\left(5 \times 10^{8}\right)$ of either Ad-GFP or Ad-GFP-S-M was given via intratracheal nebulization on day 13 after MCT, 

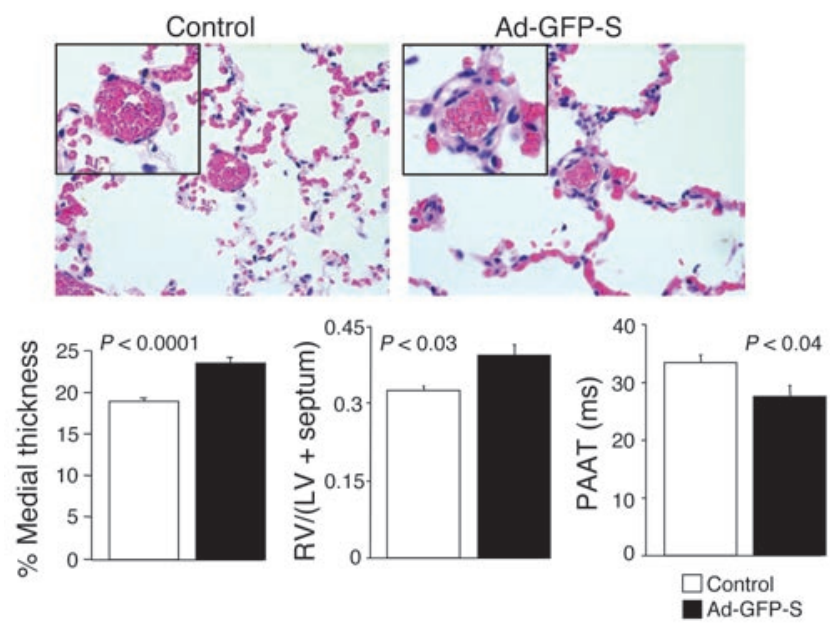

Figure 8

Exogenous WT survivin, delivered by Ad-GFP-S in normal rats, induces PAH and medial hypertrophy, within 2 weeks from infection. Magnification, $\times 10$.

as previously described (9). All rats underwent hemodynamic and echocardiography studies, and all are included in the mean data presented.

In the prevention protocol, rats received the gene therapy at the same time as MCT, i.e., day 1 . Rats ( $n=6$ per group) were randomized in MCT, MCT+Ad-GFP, and MCT+Ad-GFP-S-M groups and were studied on days 21 and 22.

In the induction protocol, normal rats were treated with inhaled saline (control, $n=6$ ) or Ad-GFP-S $(n=6)$ and were studied on days 14 and 15, in order to determine whether WT survivin would induce PAH.

Echocardiography and hemodynamics. RV free wall thickness and PA Doppler signals were measured in the parasternal short axis view using a Sonos 5500 Echo machine with $15-\mathrm{MHz}$ probes (Philips). Rats were then anesthetized with ketamine (60 mg/kg i.p.) and xylazine $(20 \mathrm{mg} / \mathrm{kg}$ i.p.) and placed on a heated table. Invasive left heart catheterization (carotid pressure and LV end-diastolic pressure [LVEDP]) and right heart catheterization were performed using high-fidelity Millar catheters (Millar Instruments Inc.); cardiac output was measured using a thermodilution probe (ADInstruments) $(8,9,26)$. PVRi was calculated as (mean PA - LVEDP)/cardiac index, and systemic vascular resistance index as (mean arterial pressure - right atrial pressure)/cardiac index.

Telemetry. A Data Sciences International system was used for telemetry (27). The implanted sensor is a fluid-filled catheter $(0.7 \mathrm{~mm}$ in diameter, 10 $\mathrm{cm}$ long) connected to a pressure transducer. Systolic pressure, diastolic pressure, and mean PA pressure were recorded for 1 minute every 4 hours (26).

Morphometric analysis of RVs and PAs. RVH was measured as RV/(LV+ septum) weight ratio at sacrifice. Lungs were inflated with formalin, fixed overnight, and embedded in paraffin. Tissue was stained with H\&E or anti$\mathrm{vWF}$ antibody. Five rats per group were studied, and from each rat at least 2 separate lung sections were examined. Resistance PAs (20-200 $\mu \mathrm{m})$ chosen randomly from low-power fields were analyzed (approximately 60 arteries per group; $2-3$ slides per rat) by 2 blinded investigators using Image-Pro Plus software (Media Cybernetics) (8, 9, 26). External diameter (ED) and medial thickness (MT) were measured, and percent medial thickness was calculated as $2 \times \mathrm{MT} \times 100 / \mathrm{ED}$. The total number of intraparenchymal PAs was also measured per low-power field (magnification, $\times 10$ ).

Cell preparation and culture. Isolated PAs (fourth to fifth division) were mechanically denuded of endothelium and digested by papain $(1 \mathrm{mg} / \mathrm{ml})$, DTT $(0.5 \mathrm{mg} / \mathrm{ml})$, collagenase $(0.6 \mathrm{mg} / \mathrm{ml})$, and BSA $(0.6 \mathrm{mg} / \mathrm{ml}$; all from
Sigma-Aldrich) for 20 minutes at $37^{\circ} \mathrm{C}$. Cells were placed in culture medium supplemented with $10 \%$ or $0.1 \%$ FBS (Sigma-Aldrich) and $1 \%$ antibiotic/antimycotic (Invitrogen Corp.) and grown in culture for 3 days at $37^{\circ} \mathrm{C}$. PASMCs in culture were exposed to Ad-GFP-S-M or Ad-GFP viruses. One hundred microliters of $5 \times 10^{9} \mathrm{PFUs}$ was used in $60-\mathrm{mm}$ plates. The exposure to virus was 6 hours, and cells were washed, kept in their respective FBS conditions, and studied 48 hours later.

Electrophysiology. Whole-cell recordings were performed using the patchclamping technique as previously described $(8,9,26)$. Cells were voltageclamped at $-70 \mathrm{mV}$, and currents were evoked by $20-\mathrm{mV}$ steps from $-70 \mathrm{mV}$ to $+70 \mathrm{mV}$ using 200 -ms pulses. Data were recorded and analyzed using pCLAMP 9 and Clampfit 9 (Axon Instruments). Whole-cell currents divided by cell capacitance gave a measure of current density.

Confocal microscopy. Imaging was performed using a Zeiss LSM 510 confocal microscope, as previously described $(8,9,26)$. Immunostaining was performed on paraffin-embedded tissue using microwave antigen retrieval and the following primary antibodies: anti-survivin (NB 500-201, 1:100; Novus Biologicals Inc.), anti-vWF and anti-smooth muscle actin (for both, 1:40; DakoCytomation), and anti-AIF (10 $\mu \mathrm{g} / \mathrm{m}$; Oncogene Research Products). Secondary antibodies were TRITC (1:200, red; Sigma-Aldrich) and FITC (1:40, green; DakoCytomation). ApopTag apoptosis detection kit (TUNEL stain, Serologicals Corp.) and the PCNA antibody (DakoCytomation) were used according to the manufacturer's instructions. BrdU was injected (100 mg/kg i.p.) 4 hours before sacrifice, and staining was performed according to the manufacturer's instructions (F. HoffmanLa Roche Ltd.). Counterstaining of nuclei with DAPI (300 nM; Invitrogen Corp.) was performed for 10 minutes at $20^{\circ} \mathrm{C}$. All were mounted with ProLong antifade compound (Invitrogen Corp.). Quantification of images (percentage of nuclei positive for TUNEL or PCNA) was done using Image-Pro Plus software by blinded investigators (26). Measurement of the mitochondrial membrane potential in live PASMCs was performed using TMRM, as previously described $(20,26)$.

Immunoblotting. Immunoblotting was performed in pooled PAs (from 3 rats per group, $25 \mu \mathrm{g}$ protein) for survivin (1:1,000; Novus Biologicals

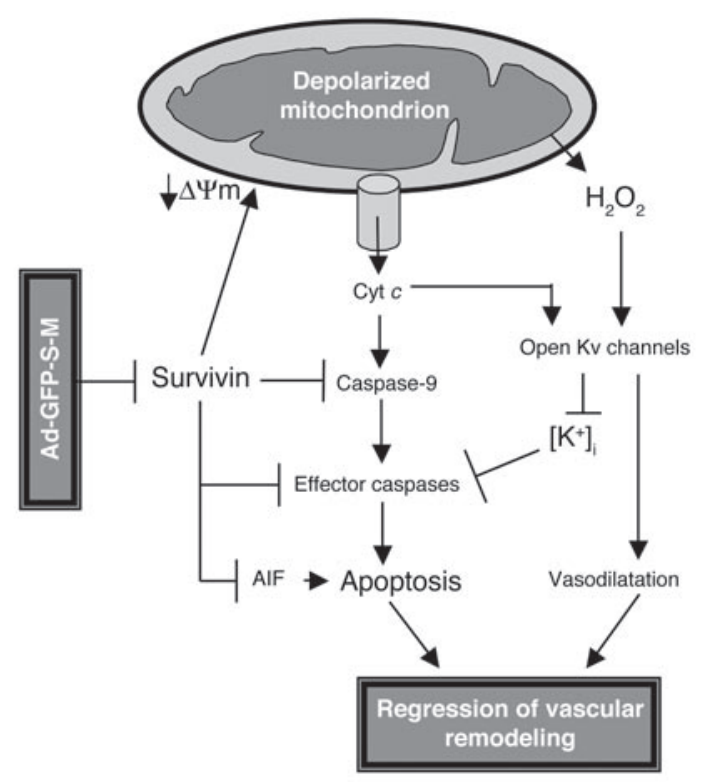

Figure 9

Schematic linking mitochondria, survivin, and $\mathrm{Kv}$ channels as potential therapeutic targets for the regression of pulmonary vascular remodeling. Cyt $c$, cytochrome $c$. 
Inc.) and in treated PASMCs (50 $\mu \mathrm{g}$ protein) for caspase assays (caspase-3, 1:500; Upstate; and caspase-9, 1:500; EMD Biosciences Inc.) as previously described (26).

Laser-capture microdissection. Lungs were inflated with and embedded in OCT, flash-frozen, and cut in 10- $\mu \mathrm{m}$ sections using a Leica CM 1850 cryostat (Leica Microsystems Inc.). HistoGene slides and dehydration/ staining reagents were used according to the manufacturer's instructions (Arcturus). Laser-capture microdissection was performed using the PixCell II system (Arcturus) as previously described $(9,26)$.

Quantitative RT-PCR. Samples were added to a microwell plate, along with TaqMan probes and reagents, and quantitative RT-PCR was performed using the ABI PRISM 7700 Sequence Detector (Applied Biosystems). Probes used included survivin, Kv1.5, bcl-2, and $18 \mathrm{~S}$ as a housekeeping gene (all from Applied Biosystems). Results for relative expression are presented as $2^{\Delta \Delta \mathrm{Ct}}$ as previously described $(9,20,26,44)$.

Statistics. Values are expressed as the mean \pm SEM. Kruskal-Wallis test or ANOVA was used as appropriate. Fisher's probable least-significant difference test was used for post hoc analysis (StatView 4.02; SAS Institute Inc.).

For survival analysis, a cohort of $20 \mathrm{MCT}$-treated rats and $20 \mathrm{Ad}$ GFP-S-M-treated rats were prospectively followed for 4 weeks and sacrificed after hemodynamic studies on day 28-30 after MCT. Spontaneous deaths were counted as events, and rats undergoing planed hemodynamic studies and sacrifice were censored. Time-to-event data were plotted using the Kaplan-Meier method, with differences evalu- ated using the log-rank test (26). A $P$ value less than 0.05 was considered statistically significant.

\section{Acknowledgments}

E.D. Michelakis and S.L. Archer are supported by the Canada Foundation for Innovation, the Alberta Heart and Stroke Foundation, the Alberta Heritage Foundation for Medical Research, the Canadian Institutes for Health Research, and the Alberta Cardiovascular and Stroke Research Centre (ABACUS). S.L. Archer is also supported by NIH grant HL071115. E.D. Michelakis is Canada Research Chair in Pulmonary Hypertension; S.L. Archer is Canada Research Chair in Translational Cardiovascular Medicine and Oxygen Sensing. M.S. McMurtry is supported by the Clinician Investigator program at the University of Alberta and a training grant from Bristol-Myers Squibb Co. D.C. Altieri is supported by NIH grants HL54131, CA78810, and CA90917.

Received for publication August 30, 2004, and accepted in revised form March 23, 2005.

Address correspondence to: Evangelos D. Michelakis, Pulmonary Hypertension Program, University of Alberta, Edmonton, Alberta T6G 2B7, Canada. Phone: (780) 407-1576; Fax: (780) 407-6032; E-mail: emichela@cha.ab.ca.
1. Archer, S., and Rich, S. 2000. Primary pulmonary hypertension: a vascular biology and translational research "work in progress". Circulation. 102:2781-2791.

2. Rubin, L.J. 2002. Therapy of pulmonary hypertension: the evolution from vasodilators to antiproliferative agents. Am. J. Respir. Crit. Care Med. 166:1308-1309.

3. Thomson, J.R., et al. 2000. Sporadic primary pulmonary hypertension is associated with germline mutations of the gene encoding BMPR-II, a receptor member of the TGF-beta family. J. Med. Genet. 37:741-745.

4. Lane, K.B., et al. 2000. Heterozygous germline mutations in BMPR2, encoding a TGF-beta receptor, cause familial primary pulmonary hypertension. The International PPH Consortium. Nat. Genet. 26:81-84.

5. Zhang, S., et al. 2003. Bone morphogenetic proteins induce apoptosis in human pulmonary vascular smooth muscle cells. Am. J. Physiol. Lung Cell. Mol. Physiol. 285:L740-L754.

6. Morrell, N.W., et al. 2001. Altered growth responses of pulmonary artery smooth muscle cells from patients with primary pulmonary hypertension to transforming growth factor-beta(1) and bone morphogenetic proteins. Circulation. 104:790-795.

7. Geraci, M.W., et al. 2001. Gene expression patterns in the lungs of patients with primary pulmonary hypertension: a gene microarray analysis. Circ. Res. 88:555-562.

8. Michelakis, E.D., et al. 2002. Dichloroacetate, a metabolic modulator, prevents and reverses chronic hypoxic pulmonary hypertension in rats: role of increased expression and activity of voltage-gated potassium channels. Circulation. 105:244-250.

9. Pozeg, Z.I., et al. 2003. In vivo gene transfer of the $\mathrm{O} 2$-sensitive potassium channel Kv1.5 reduces pulmonary hypertension and restores hypoxic pulmonary vasoconstriction in chronically hypoxic rats. Circulation. 107:2037-2044.

10. Yuan, J.X., et al. 1998. Dysfunctional voltage-gated $\mathrm{K}+$ channels in pulmonary artery smooth muscle cells of patients with primary pulmonary hypertension. Circulation. 98:1400-1406.
11. Archer, S.L., et al. 1998. Molecular identification of the role of voltage-gated $\mathrm{K}+$ channels, Kv1.5 and $\mathrm{Kv} 2.1$, in hypoxic pulmonary vasoconstriction and control of resting membrane potential in rat pulmonary artery myocytes. J. Clin. Invest. 101:2319-2330.

12. Platoshyn, O., et al. 2000. Sustained membrane depolarization and pulmonary artery smooth muscle cell proliferation. Am. J. Physiol. Cell Physiol. 279:C1540-C1549.

13. Yu, Y., et al. 2004. Enhanced expression of transient receptor potential channels in idiopathic pulmonary arterial hypertension. Proc. Natl. Acad. Sci. U. S. A. 101:13861-13866.

14. Remillard, C.V., and Yuan, J.X. 2004. Activation of $\mathrm{K}+$ channels: an essential pathway in programmed cell death. Am. J. Physiol. Lung Cell. Mol. Physiol. 286:L49-L67.

15. Salvesen, G.S., and Duckett, C.S. 2002. IAP proteins: blocking the road to death's door. Nat. Rev. Mol. Cell Biol. 3:401-410.

16. Altieri, D.C. 2003. Validating survivin as a cancer therapeutic target. Nat. Rev. Cancer. 3:46-54.

17. Dohi, T., Beltrami, E., Wall, N.R., Plescia, J., and Altieri, D.C. 2004. Mitochondrial survivin inhibits apoptosis and promotes tumorigenesis. J. Clin. Invest. 114:1117-1127. doi:10.1172/ JCI200422222.

18. Blanc-Brude, O.P., et al. 2002. Inhibitor of apoptosis protein survivin regulates vascular injury. Nat. Med. 8:987-994.

19. Weir, E.K., and Archer, S.L. 1995. The mechanism of acute hypoxic pulmonary vasoconstriction: the tale of two channels. FASEB J. 9:183-189.

20. Michelakis, E.D., et al. 2002. Diversity in mitochondrial function explains differences in vascular oxygen sensing. Circ. Res. 90:1307-1315.

21. Caouette, D., Dongmo, C., Berube, J., Fournier, D., and Daleau, P. 2003. Hydrogen peroxide modulates the Kv1.5 channel expressed in a mammalian cell line. Naunyn Schmiedebergs Arch. Pharmacol. 368:479-486.

22. Wang, D., et al. 1996. NADPH-oxidase and a hydrogen peroxide-sensitive $\mathrm{K}+$ channel may function as an oxygen sensor complex in airway chemoreceptors and small cell lung carcinoma cell lines. Proc.
Natl. Acad. Sci. U. S. A. 93:13182-13187.

23. Burke, T., and Wolin, M. 1987. Hydrogen peroxide elicits pulmonary arterial relaxation and guanylate cyclase activation. Am. J. Physiol. 252:H721-H732.

24. Duchen, M.R. 1999. Contributions of mitochondria to animal physiology: from homeostatic sensor to calcium signalling and cell death. J. Physiol. 516:1-17.

25. Jones, J.E., et al. 2002. Serial noninvasive assessment of progressive pulmonary hypertension in a rat model. Am. J. Physiol. Heart Circ. Physiol. 283:H364-H371.

26. McMurtry, M.S., et al. 2004. Dichloroacetate prevents and reverses pulmonary hypertension by inducing pulmonary artery smooth muscle cell apoptosis. Circ. Res. 95:830-840.

27. Hess, P., Clozel, M., and Clozel, J.P. 1996. Telemetry monitoring of pulmonary arterial pressure in freely moving rats. J. Appl. Physiol. 81:1027-1032.

28. Yuan, X.J., Wang, J., Juhaszova, M., Gaine, S.P., and Rubin, L.J. 1998. Attenuated K+ channel gene transcription in primary pulmonary hypertension [letter]. Lancet. 351:726-727.

29. Daugas, E., et al. 2000. Apoptosis-inducing factor (AIF): a ubiquitous mitochondrial oxidoreductase involved in apoptosis. FEBS Lett. 476:118-123.

30. Platoshyn, O., Zhang, S., McDaniel, S.S., and Yuan, J.X. 2002. Cytochrome c activates $\mathrm{K}+$ channels before inducing apoptosis. Am. J. Physiol. Cell Physiol. 283:C1298-C1305.

31. Zhao, Y.D., Campbell, A.I., Robb, M., Ng, D., and Stewart, D.J. 2003. Protective role of angiopoietin1 in experimental pulmonary hypertension. Circ. Res. 92:984-991.

32. Molteni, A., Ward, W.F., Ts'ao, C.H., Port, C.D., and Solliday, N.H. 1984. Monocrotaline-induced pulmonary endothelial dysfunction in rats. Proc. Soc. Exp. Biol. Med. 176:88-94.

33. Arcot, S.S., Lipke, D.W., Gillespie, M.N., and Olson, J.W. 1993. Alterations of growth factor transcripts in rat lungs during development of monocrotaline-induced pulmonary hypertension. Biochem. Pharmacol. 46:1086-1091.

34. Cowan, K.N., et al. 2000. Complete reversal of fatal pulmonary hypertension in rats by a serine elastase inhibitor. Nat. Med. 6:698-702. 
35. Nishimura, T., et al. 2003. Simvastatin rescues rats from fatal pulmonary hypertension by inducing apoptosis of neointimal smooth muscle cells. Circulation. 108:1640-1645.

36. Abe, K., et al. 2004. Long-term treatment with a Rho-kinase inhibitor improves monocrotalineinduced fatal pulmonary hypertension in rats. Circ. Res. 94:385-393.

37. Rich, S., Kaufmann, E., and Levy, P.S. 1992. The effect of high doses of calcium-channel blockers on survival in primary pulmonary hypertension. N. Engl.J. Med. 327:76-81.
38. Duchen, M.R. 2000. Mitochondria and $\mathrm{Ca}(2+)$ in cell physiology and pathophysiology. Cell Calcium. 28:339-348.

39. Wang, H., et al. 2002. HERG K+ channel, a regulator of tumor cell apoptosis and proliferation. Cancer Res. 62:4843-4848.

40. O'Connor, D.S., et al. 2000. Regulation of apoptosis at cell division by $\mathrm{p} 34 \mathrm{cdc} 2$ phosphorylation of survivin. Proc. Natl. Acad. Sci. U. S. A. 97:13103-13107.

41. Xu, Y., Fang, F., Ludewig, G., Jones, G., and Jones, D. 2004. A mutation found in the promoter region of the human survivin gene is correlated to overexpression of survivin in cancer cells. DNA Cell Biol. 23:527-537.

42. Voelkel, N.F., et al. 1998. Primary pulmonary hypertension between inflammation and cancer. Chest. 114(Suppl.):225S-230S

43. Budhiraja, R., Tuder, R.M., and Hassoun, P.M. 2004. Endothelial dysfunction in pulmonary hypertension. Circulation. 109:159-165.

44. Michelakis, E.D., et al. 2002. O2 sensing in the human ductus arteriosus: regulation of voltagegated $\mathrm{K}+$ channels in smooth muscle cells by a mitochondrial redox sensor. Circ. Res. 91:478-486. 